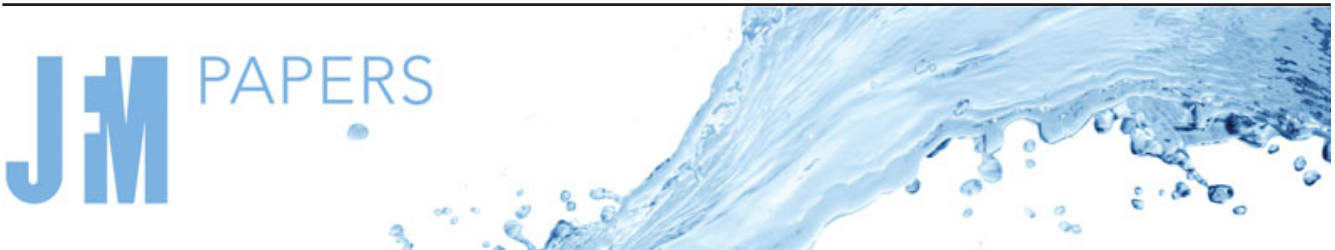

\section{The self-similar stratified inner-shelf response to transient rip-current-induced mixing}

\author{
Derek J. Grimes $^{1, \dagger}$ and Falk Feddersen ${ }^{1}$ \\ ${ }^{1}$ Scripps Institution of Oceanography, University of California San Diego, La Jolla, CA 92037, USA
}

(Received 7 October 2020; revised 22 December 2020; accepted 9 February 2021)

The stratified inner-shelf response to surf-zone-generated transient rip currents (TRC) is examined using idealized simulations with uniform initial thermal stratification $\mathrm{d} T_{0} / \mathrm{d} z$, with initial temperature $T_{0}$ and vertical coordinate $z$, or initial squared buoyancy frequency $N_{0}^{2}$, varying from unstratified to highly stratified $\left(0.75^{\circ} \mathrm{C} \mathrm{m}-1\right)$. The TRC-induced depth-integrated cross-shore eddy kinetic energy flux is independent of $\mathrm{d} T_{0} / \mathrm{d} z$ and decays to near zero within $4 L_{S Z}$ (surf-zone width $L_{S Z}=100 \mathrm{~m}$ ). Cross-shore inhomogeneous TRC mixing causes shoreward broadening isotherms, driving a near-field inner-shelf overturning circulation and a far-field geostrophic along-shore velocity that strengthen with $\mathrm{d} T_{0} / \mathrm{d} z$. TRC mixing mostly $(90 \%)$ increases background potential energy (BPE) and also available (APE, $10 \%$ ) potential energy, driving inner-shelf mean circulation. The specific BPE zero-crossing depth $d_{s}$ collapses the near-field 3-layer cross-shore velocity, using an intrusive gravity current scaling $\left(d_{s} N_{0}\right)$. The approximately steady exchange flow exports low-stratification fluid $\left(N / N_{0} \approx 1 / 2\right)$ at a depth $z / d_{s} \approx-1$, re-supplying the TRC region with stratified fluid from above/below, similar to localized mixing laboratory experiments. Offshore of $\approx 5 L_{S Z}$, a self-similar far-field intrusion with characteristic isotherm slope $d_{s} / L_{R}$ (Rossby deformation radius $L_{R} \sim d_{S} f / N_{0}$ ) is in approximate geostrophic balance with the non-dimensional along-shore velocity. Inner-shelf near-field and far-field horizontal length scales vary as $x / d_{s}$ and $x / L_{R}$, respectively. The length scale $d_{s}$ is related to the work performed by TRC mixing using an idealized well-mixed wedge geometry. Idealized analytical scalings are qualitatively consistent with modelled BPE and APE distributions. Thus, the self-similar stratified inner-shelf response to TRC-driven mixing depends on key dimensional parameters $N_{0}$ and $d_{s}$.

Key words: baroclinic flows, shallow water flows, surface gravity waves

$†$ Email address for correspondence: dgrimes@ucsd.edu

(C) The Author(s), 2021. Published by Cambridge University Press. This is an Open Access article, distributed under the terms of the Creative Commons Attribution licence (http://creativecommons.org/ licenses/by/4.0/), which permits unrestricted re-use, distribution, and reproduction in any medium, provided the original work is properly cited. 


\section{D.J. Grimes and F. Feddersen}

\section{Introduction}

The inner shelf is influenced by myriad processes over broad spatial and temporal scales (100 m-10 km and $1 \mathrm{~h}-1$ month, Lentz \& Fewings 2012; Brink 2016) that regulate nutrient cycling and larval recruitment (Washburn \& McPhee-Shaw 2013; Morgan et al. 2018) and coastal water quality (Boehm et al. 2017). On sub-tidal time scales, inner-shelf stratification and circulation are modified by wind-driven mixing and cross-shore transport (upwelling/downwelling) (e.g. Lentz \& Fewings 2012), and modulated by tidal mixing (e.g. Castelao et al. 2010). On both diurnal and longer time scales, solar heating and surface buoyancy fluxes regulate inner-shelf stratification and circulation (e.g. Monismith et al. 2006; Molina et al. 2014). Internal waves and tides induce cross-shelf exchange and vertical mixing on time scales ranging from $10 \mathrm{~min}$ to $12 \mathrm{~h}$ (e.g. Walter et al. 2012; Sinnett et al. 2018). On relatively fast time scales ( $<1 \mathrm{~h})$, strong and concentrated offshore directed rip currents enhance exchange between the surf zone and inner shelf (Brown et al. 2009; Clark, Feddersen \& Guza 2010; Reniers et al. 2010). After several hours (> $6 \mathrm{~h}$ and $<48 \mathrm{~h}$ ), transient rip-current (TRC) enhanced vertical mixing on the stratified inner shelf drives inner-shelf circulation (Kumar \& Feddersen 2017b,c). However, the stratified inner-shelf response to transient rip currents remains poorly understood.

Rip currents are surf-zone-generated strong offshore flows extending onto the inner shelf and have various classifications (cf. Castelle et al. 2016). On along-shore uniform coasts, TRC are ephemeral with no preferred along-shore formation site (Dalrymple et al. 2011). TRC generation is due to the coalescence of surf-zone vorticity forced by short-crested breaking waves (Peregrine 1998; Johnson \& Pattiaratchi 2006; Feddersen 2014). In contrast to TRCs, bathymetrically controlled rip currents develop through the coupling of shoaling waves and surf-zone currents with underlying bathymetry, and preferentially form in rip channels (e.g. MacMahan et al. 2010). In the surf zone, TRCs are predominately barotropic (depth uniform), with strength and occurrence varying with incident wave characteristics, particularly wave directional spread $\sigma_{\theta}$, and beach slope $\beta$ (e.g. Johnson $\&$ Pattiaratchi 2006). In depth-averaged TRC-resolving simulations, TRC-driven barotropic exchange occurs within approximately $2 L_{S Z}$ to $4 L_{S Z}$ of shore ( $L_{S Z}$ is the surf-zone width) and is self-similar, depending on incident wave and beach characteristics (Suanda \& Feddersen 2015). In a depth-resolving model, TRC-induced eddies enhance exchange across the unstratified inner shelf relative to the Stokes drift/undertow exchange in non-TRC-resolving (i.e. phase-averaged) simulations (Kumar \& Feddersen 2017a).

The inner shelf is often stratified, and TRC-enhanced vertical mixing reduces inner-shelf stratification within a few surf-zone widths of shore $\left(\approx 4 L_{S Z}\right)$, causing shoreward broadening of modelled isopycnals and increasing the local depth integrated potential energy (Kumar \& Feddersen 2017b). The modelled cross-shore sloping isopycnals drive a baroclinic cross-shore exchange flow that transports surf-zone released passive tracer within a low-stratification offshore intrusion up to $8 L_{S Z}$ across the inner shelf (Kumar $\&$ Feddersen 2017c). In contrast, unstratified simulations had tracer confined to shore and vertically well mixed. Thus, baroclinic exchange extends tracer spreading well beyond the region directly affected by TRCs. However, as Kumar \& Feddersen $(2017 b, c)$ only considered a single non-zero spatially uniform thermal stratification of $\mathrm{d} T_{0} / \mathrm{d} z=$ $0.25^{\circ} \mathrm{C} \mathrm{m}^{-1}$, characteristic of summer-autumn in Southern California, the stratification dependence of TRC-driven mixing and exchange across the inner shelf could not be determined.

The inhomogeneous TRC-mixing-induced circulation is analogous to other geophysical flows driven by spatial mixing gradients. In a localized near-surface mixing experiment, 


\section{Self-similar inner-shelf response to transient rip currents}

Maxworthy \& Monismith (1988) found that baroclinic flow away from the mixing region scales similar to an intrusive gravity current velocity $\mathcal{U} \sim N d$ with ambient buoyancy frequency $N=\left(-g \rho^{-1} \partial \rho / \partial z\right)^{1 / 2}$, density $\rho$, constant gravitational acceleration $g$, and mixed layer depth $d$ (e.g. Bolster, Hang \& Linden 2008). Experiments were initialized from rest and the mixed layer depth grew until the gravity current transport balanced entrainment at the base of the mixing region. Based on well-defined scalings for grid-generated turbulence (Hopfinger \& Toly 1976), the equilibrium mixed layer depth roughly scaled as $d \sim N^{-1 / 2}$ (Maxworthy \& Monismith 1988), the Ozmidov length scale in this context (Maderich, Van Heijst \& Brandt 2001). Here, the bottom is sloping and TRC-induced mixing occurs over much of the water column and has a cross-shore decay scale, precluding a theoretical deduction of the stratified inner-shelf response.

On long time scales, Coriolis effects may deflect the offshore propagating intrusion (e.g. Davies et al. 1991; Thomas \& Linden 2007), potentially modulating intrusion growth. Kumar \& Feddersen $(2017 b)$ found that $>4.5 L_{S Z}$ offshore, sloping isopycnals approached thermal wind balance after $42-48 \mathrm{~h}\left(\approx 2 f^{-1}\right)$ with $f$ the Coriolis frequency. In wind-driven upwelling/downwelling regimes, geostrophic interior (away from boundaries) isopycnal slope $(\mathrm{d} z / \mathrm{d} x)$ may vary weakly with depth and is characterized by the local Rossby deformation radius $\left(L_{R}=N H / f\right)$, or $\mathrm{d} z / \mathrm{d} x \sim H / L_{R}=f / N$, with depth scale $H$ (e.g. Lentz $\&$ Chapman 2004). Mid-shelf tidal mixing also induces sloping isopycnals that tend to broaden toward shallow water with aspect ratio scaling $f / N$ and form an offshore mixed fluid intrusion (e.g. Garrett \& Loder 1981; van Heijst 1986). However, the relevant depth scale $H$ and cross-shore region where geostrophic scalings apply to the case of TRC-induced mixing are unknown.

Natural variability of inner-shelf stratification, owing to many contributing processes, complicates the use of field observations. For example, internal waves and diurnal solar heating/cooling can significantly modify vertical and cross-shore buoyancy gradients over time scales of hours (e.g. Omand et al. 2011; Sinnett et al. 2018; Grimes et al. 2020a). Such fast variations in stratification, relative to that of individual TRC-induced eddies $(O(1 \mathrm{~h}))$, are likely to interact with and modulate TRC exchange. Idealized modelling studies circumvent these difficulties and allow processes to be isolated. Based on an ensemble of idealized simulations that included a zero-net diurnal heating/cooling cycle, diurnal-averaged TRC exchange was similar with and without surface heat flux and dominant relative to diurnal-averaged thermally driven exchange (Grimes, Feddersen \& Kumar 2020b).

The inner-shelf stratification and circulation response to surf-zone and TRC-induced mixing are determined using idealized simulations with wave and surf-zone TRC forcing only. A spatially uniform initial thermal stratification $\mathrm{d} T_{0} / \mathrm{d} z(\S 2.1)$ is varied to determine the relevant dimensional scales and non-dimensional parameters describing the inner-shelf response. Inner-shelf response is quantified using along-shore-averaged and intermediate time scale (from 24-48 h) mean statistics (\$2.3), when the baroclinic exchange flow is approximately steady. In $\S 3$, the effects of initial stratification on inner-shelf mean and eddy statistics are described qualitatively. In $\S 4$, energetics analysis is used to determine the sensitivity of TRC-induced eddy statistics to varying initial stratification and isolate a vertical length scale $\left(d_{s}\right)$ quantifying the potential energy response to mixing. In $\S 5$, dimensional quantities including $d_{s}$ are used to derive and evaluate non-dimensional stratification, circulation and potential energy scalings. Modelled $d_{s}$ variation is related to the initial stratification and the surf-zone to the inner-shelf eddy kinetic energy flux in $\S \S 6.1$ and 6.2 using an idealized schematic representation of TRC mixing effects on the stratified innershelf derived in Appendices A and B. These results are extrapolated to 


\section{D.J. Grimes and F. Feddersen}

varying incident wave properties using existing TRC-induced exchange velocity scalings in $\S 6.3$. Lastly, results are summarized and contextualized in $\S 7$.

\section{Methods}

\subsection{Modelling}

The COAWST modelling framework is used here (Warner et al. 2010) with methods similar to those used by Kumar \& Feddersen $(2017 a, b, c)$ and Grimes et al. (2020b). The hydrodynamic regional ocean modelling system (ROMS, Shchepetkin \& McWilliams 2005) is one-way coupled (wave effects on currents) to the spectral simulating waves nearshore (SWAN, Booij, Ris \& Holthuijsen 1999). ROMS is also supplied with surf-zone breaking wave rotational body forcing from the phase-resolving wave model funwaveC (Kumar \& Feddersen 2017a), which generates surf-zone vertical vorticity and spontaneous formation of TRCs, in a manner consistent with observations (Feddersen 2014). One-way coupled SWAN does not account for current effects on waves (CEW) and potential rip-current-induced wave refraction, which can modify rip-current circulation (Yu \& Slinn 2003). In general, CEW depend on the ratio of the cross-shore Eulerian velocity $u$ to the barotropic wave speed $c_{g}=(g h)^{1 / 2}$ (Mei 1992), which is of $O\left(10^{-2}\right.$ ) on the inner shelf in the present simulations (cf. $\S 5 c$ in Kumar \& Feddersen 2017a). Additionally, the magnitude and cross-shore distribution of root-mean-square vertical vorticity are similar for both funwaveC, which includes CEW, and COAWST simulations (cf. figure 5 in Kumar \& Feddersen 2017a), suggesting modelled rip-current statistics are well represented.

The model cross-shore $(x)$ domain extends $L_{x}=5 \mathrm{~km}$ offshore (figure $1 a$ ), with $x=0$ at the shoreline and positive onshore. Close to shore, $x \geq-250 \mathrm{~m}$, bathymetry $h(x)$ has constant slope $\beta_{S Z}=0.025$ that transitions quadratically to $\beta_{I S}=0.004$ offshore of $x=-1000 \mathrm{~m}$ (black, figure $1 a$ ). The cross-shore grid resolution is fixed at $\Delta x=1.25 \mathrm{~m}$ for $x>-690 \mathrm{~m}$ and telescopes smoothly to $\Delta x=200 \mathrm{~m}$ at $x=-5 \mathrm{~km}$. The domain is along-shore $(y)$ uniform and periodic, spanning $L_{y}=1 \mathrm{~km}$. The vertical coordinate $z=0$ at the mean water level with positive upwards. Fifteen ROMS terrain following vertical $\sigma$ coordinates are used, and near-surface resolution is slightly increased offshore (grey, figure $1 a$ ).

Tracer vertical eddy diffusivity $K_{V}$ is determined using the two equation $k-\epsilon$ turbulence closure for the turbulent kinetic energy $(k)$ and rate of dissipation $(\epsilon)$ with Kantha \& Clayson (1994) stability functions (Warner, Geyer \& Lerczak 2005). In the surf-zone, $15 \%$ of wave energy dissipation is supplied as surface turbulent kinetic energy flux, consistent with surf-zone observations (Feddersen 2012) and previous modelling studies (e.g. Rodriguez, Giddings \& Kumar 2018). The background horizontal diffusivity $K_{H}=0.01 \mathrm{~m}^{2} \mathrm{~s}^{-1}$ is increased $20 \times$ within the surf zone, to account for unresolved bore/roller dispersion, but remains $\approx 5 \times$ smaller than observed surf-zone rotational eddy-driven stirring (e.g. Clark et al. 2010; Clark, Feddersen \& Guza 2011). A spatially uniform quadratic bottom drag $C_{d}=0.0025$ and Coriolis parameter $f=7.7 \times 10^{-5} \mathrm{~s}^{-1}$ are used. Simulated wave conditions are typical of Southern California in summer-autumn with normally incident $H_{s z} \approx 1 \mathrm{~m}$ significant wave height at breaking $x=-100 \mathrm{~m}$ (figure $1 b$ ), peak period $T_{p}=10 \mathrm{~s}$ and directional spread $\sigma_{\theta}=10^{\circ}$. The surf-zone width $L_{S Z}=100 \mathrm{~m}$ is used as a characteristic cross-shore length scale (figure $1 b$ ).

The inner shelf can have a range of stratifications (e.g. Lentz 2001) from uniform stratification (e.g. Feddersen et al. 2020) to an approximate two layer structure (e.g. Grimes et al. 2020a). Here, a series of 18 simulations are performed varying the initial stratification $\mathrm{d} T_{0} / \mathrm{d} z=\{0,0.0125,0.025,0.05: 0.05: 0.75\}^{\circ} \mathrm{C} \mathrm{m}^{-1}$ to evaluate 


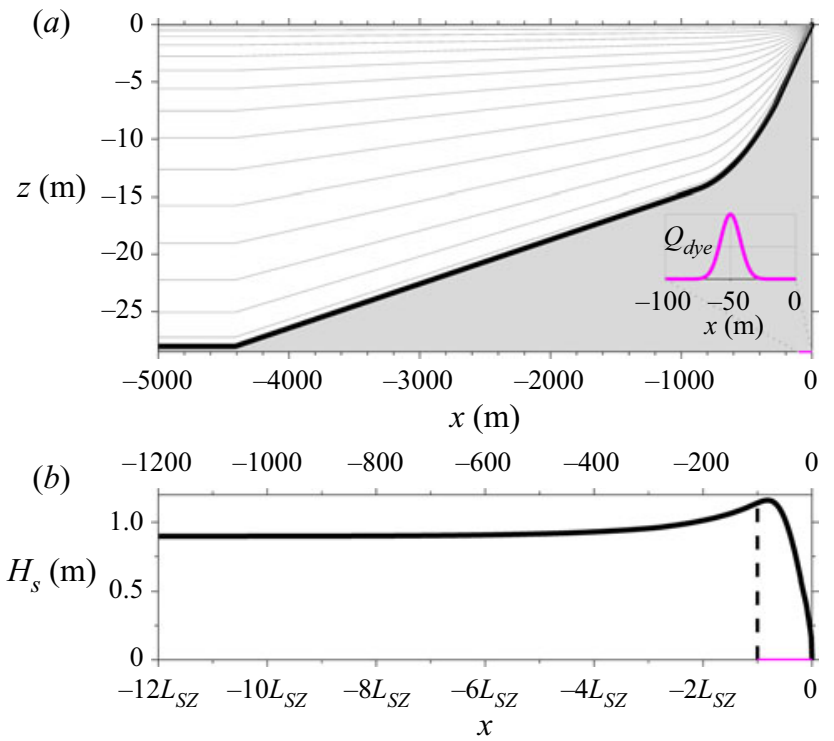

Figure 1. (a) Cross-shore $(x)$ model domain with depth $z=-h(x)$ in black and ROMS $\sigma$-layers in grey. The inset illustrates the along-shore uniform $50 \mathrm{~m}$ wide Gaussian tracer bottom flux $Q_{d y e}$ centred on $x=-50 \mathrm{~m}$ or $x=-L_{S Z} / 2$. (b) SWAN significant wave height $H_{S}(x)$ with the surf-zone boundary, $x=-L_{S Z}=-100 \mathrm{~m}$, indicated by vertical dashed black line. The lower $x$-axis of $(b)$ is measured in surf-zone width $L_{S Z}$ with the surf-zone and tracer release region from inset $(a)$ indicated by magenta coloured $x$-axis. Note the cross-shore ranges differ in $(a)$ and $(b)$.

how inner-shelf stratification affects TRC mixing and induced inner-shelf circulation. The $48 \mathrm{~h}$ long simulations are initialized from rest, with spatially uniform and constant initial stratification $\mathrm{d} T_{0} / \mathrm{d} z$,

$$
T(z, t=0) \equiv T_{0}(z)=T_{r e f}+z \frac{\mathrm{d} T_{0}}{\mathrm{~d} z}
$$

with surface reference temperature $T_{r e f}=20^{\circ} \mathrm{C}$. A linear temperature dependent equation of state is used, with initial condition,

$$
\rho(z, t=0) \equiv \rho_{0}(z)=\varrho\left[1-\alpha\left(T_{0}(z)-T_{\varrho}\right)\right],
$$

with constant reference density $\varrho=1027 \mathrm{~kg} \mathrm{~m}^{-3}$, and linearized about $T_{\varrho}=20^{\circ} \mathrm{C}$, giving a constant thermal expansion coefficient $\alpha=1.7 \times 10^{-4} \mathrm{C}^{-1}$. The initial buoyancy frequency $N_{0}=\left(g \alpha \mathrm{d} T_{0} / \mathrm{d} z\right)^{1 / 2}$ varies over $\{0,4.6,6.5,9.1,12.9, \ldots, 35.3\} \times 10^{-3} \mathrm{~s}^{-1}$. Offshore boundary $(x=-5 \mathrm{~km})$ temperature is clamped to the initial condition $T_{0}(z)$. A zero mass flux offshore boundary condition is applied at each $z$-level. The surface and bottom boundaries are adiabatic and surface stress is zero, i.e. no wind or surface heat fluxes. Similar to Grimes et al. (2020b), a series of 4 mid-surf-zone passive tracer releases occur at $6 \mathrm{~h}$ intervals in a $50 \mathrm{~m}$ wide cross-shore Gaussian distributed and along-shore uniform strip (inset of figure 1a). Dilution of shoreline released tracers affects coastal water quality (e.g. Boehm et al. 2017), and tracer mass budgets are a useful measure of cross-shore exchange (e.g. Hally-Rosendahl et al. 2015). 


\section{D.J. Grimes and F. Feddersen}
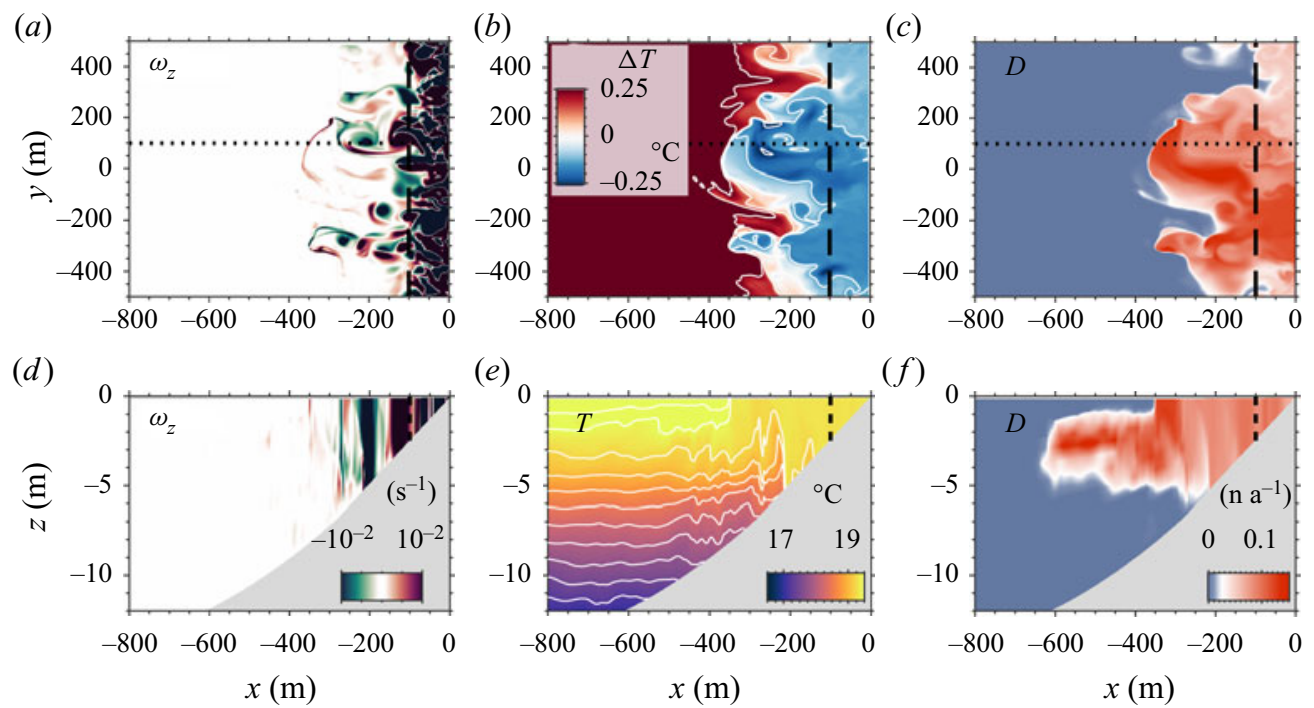

(e)
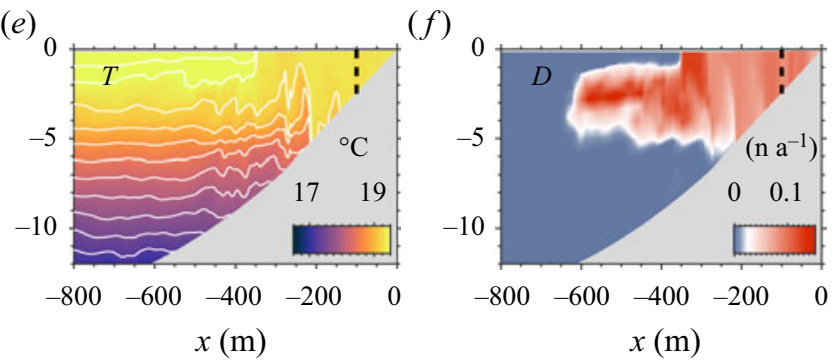

Figure 2. Model time $t=12 \mathrm{~h} ;(a, d)$ vertical component of relative vorticity $\omega_{z},(b)$ surface temperature anomaly $\Delta T$ relative to the $x=-150 \mathrm{~m}$ along-shore mean, $(e)$ sub-surface temperature $T$ and $(c, f)$ tracer $D$. Surface panels $(a)-(c)$ are functions of $(x, y)$ and sub-surface panels $(d)-(f)$ are functions of $(x, z)$ at $y=100 \mathrm{~m}$, as indicated with black dotted line in $(a)-(c)$. In $(c, f)$, mid-surf-zone released tracer $D$ has evolved for $6 \mathrm{~h}$ (see figure 1(a) inset, and Grimes et al. (2020b) for more details). The $L_{S Z}=100 \mathrm{~m}$ wide surf-zone is delimited with vertical dashed black line, and in $(d)-(f)$ the bottom is grey.

\subsection{Description of model snapshot}

An example snapshot from the $\mathrm{d} T_{0} / \mathrm{d} z=0.25^{\circ} \mathrm{C} \mathrm{m}^{-1}$ simulation is presented to motivate the averaging methods used in analysing the range of $\mathrm{d} T_{0} / \mathrm{d} z$ considered. At model time $t=12 \mathrm{~h}$, surf-zone-generated TRC-induced eddies occupy the region $x>-4 L_{S Z}$ (figure $2 a$ ), e.g. at $\left(x=-2 L_{S Z}, y=100 \mathrm{~m}\right)$ in figures $2(a)$ and $2(d)$, with peak vertical vorticity of $O(100 f)$. Near the surf zone, TRC eddies are barotropic and temperature $T$ and surf-zone $t=6 \mathrm{~h}$ released tracer $D$ are vertically well mixed, e.g. $x \geq-2.5 L_{S Z}$ in figure $2(e, f)$. As such, the complex swirling and stirring patterns in surface $T$ and $D$ are representative of the depth-averaged TRC eddy-induced variability for $x>-3 L_{S Z}$ (figure $2 b, c$ ).

In depth-averaged models, TRC are non-divergent horizontal eddies with vertical vorticity. In depth-resolving models, inner-shelf TRC motions have vertical structure and are horizontally divergent, inducing vertical velocity. For example, offshore of $3 L_{S Z}$, inner-shelf TRC-induced eddies have increased vertical structure relative to near surf zone and with filamentous vorticity features (e.g. feature at $x=-3.5 L_{S Z}$ in figures $2 a$ and $2 d$ ). Along $y=100 \mathrm{~m}$, vertical isotherm displacements of $>1$ $\mathrm{m}$ that vary with depth occur out to $x=-6 L_{S Z}$ (figure $2 e$ ). The largest vertical displacements are offshore of a strong anticyclonic eddy (figure $2 d$ ), linking TRC-induced eddies to development of $T$ inversions and mixing. The TRC-enhanced vertical mixing generates along-shore-averaged cross-shore temperature gradients and baroclinic inner-shelf circulation (e.g. Kumar \& Feddersen 2017b). Tracer $D$ was released 6 h prior and is transported offshore of the TRC dominated region $\left(x>-4 L_{S Z}\right)$ in a sub-surface intrusion by an along-shore-averaged baroclinic $(x, z)$-exchange flow (figure $2 f$ ). Sub-surface tracer export is the hallmark of the TRC-induced baroclinic exchange mechanism (cf. Kumar \& Feddersen 2017c; Grimes et al. 2020b), and a 


\section{Self-similar inner-shelf response to transient rip currents}

primary focus of this work is quantifying and scaling the vertical thickness, strength and cross-shore structure of the circulation with varying stratification.

\subsection{Averaging and analysis}

To analyse TRC-induced eddy variability separately from the effects of enhanced vertical mixing on mean inner-shelf temperature and circulation, dynamical variables are decomposed into along-shore mean and perturbation components,

$$
c(x, y, z, t)=\bar{c}(x, z, t)+c^{\prime}(x, y, z, t),
$$

where the along-shore mean of generic variable $c$ is,

$$
\bar{c}(x, z, t)=L_{y}^{-1} \int_{0}^{L_{y}} c\left(x, y^{\prime}, z, t\right) \mathrm{d} y^{\prime},
$$

and $c^{\prime}$ is the along-shore perturbation or eddy component. Following a $<6 \mathrm{~h}$ spin-up period, the simulated inner-shelf TRC eddy statistics approach stationarity with low-frequency variability, analogous to surf-zone very-low-frequency Eulerian current variability (e.g. Elgar \& Raubenheimer 2020). The along-shore mean statistics evolve in time due to irreversible mixing (e.g. Grimes et al. 2020b). Aspects of the $\S \S 3$ and 4 analysis use a time average from $t=24-48 \mathrm{~h}$,

$$
\langle c\rangle(x, z)=(24 \mathrm{~h})^{-1} \int_{24 \mathrm{~h}}^{48 \mathrm{~h}} c\left(x, z, t^{\prime}\right) \mathrm{d} t^{\prime},
$$

to diagnose stratified inner-shelf response to TRCs. The chosen time-averaging period begins after and spans an inertial period $\left(>f^{-1} \approx 23 \mathrm{~h}\right.$ ). After $\approx 18 \mathrm{~h}$, the circulation and stratification within $x \gtrsim-600 \mathrm{~m}$ evolve slowly such that over the $24-48 \mathrm{~h}$ averaging period they can be considered quasi-steady. Salient features and scalings are similar for shorter averaging periods (with duration $>6 \mathrm{~h}$ ). Hereafter, 'mean' will refer to both along-shore average (2.4) and 24-48 h time average (2.5), unless otherwise noted.

\section{Results: inner-shelf response to wave and TRC forcing}

\subsection{Mean inner-shelf response with varying stratification}

A qualitative picture of how varying stratification affects TRC-induced inner-shelf mixing, temperature and circulation is revealed by comparing the mean, along-shore (2.4) and 24-48 h time-averaged (2.5), (x, z)-structure of tracer vertical eddy diffusivity $\left\langle\bar{K}_{V}\right\rangle$, temperature $\langle\bar{T}\rangle$, streamfunction $\langle\bar{\Psi}\rangle$ and along-shore velocity $\langle\bar{v}\rangle$ at three levels of stratification $\mathrm{d} T_{0} / \mathrm{d} z=\{0.025,0.1,0.25\}^{\circ} \mathrm{C} \mathrm{m}^{-1}$ (figure 3). In the region significantly affected by TRC-induced eddy activity, $x>-4 L_{S Z}$ and denoted the TRC region, $\left\langle\bar{K}_{V}\right\rangle$ is elevated, increasing from $10^{-6} \mathrm{~m}^{2} \mathrm{~s}^{-1}$ offshore to $10^{-2} \mathrm{~m}^{2} \mathrm{~s}^{-1}$ in the surf zone (figure $3 a$ ). Increasing stratification (left to right in figure 3) decreases the inner-shelf $\left(x<-L_{S Z}\right)\left\langle\bar{K}_{V}\right\rangle$ (figure $3 a-c)$, confining the elevated mixing region to a smaller $(x, z)$-area. The elevated inner-shelf $\left\langle\bar{K}_{V}\right\rangle$ is due to both temperature (density) inversions and shear-driven mixing. The $\left\langle\bar{K}_{V}\right\rangle$ reduction with $\mathrm{d} T_{0} / \mathrm{d} z$ is consistent with a suppression of vertical mixing by strong stratification (e.g. Burchard \& Bolding 2001; Kumar \& Feddersen 2017b). Reduced TRC region stratification is evidenced by shoreward broadening isotherms and sub-surface intrusion of mixed fluid extending offshore and centred on $z \approx-4 \mathrm{~m}$ (e.g. figure $3 d$ ). 


\section{D.J. Grimes and F. Feddersen}
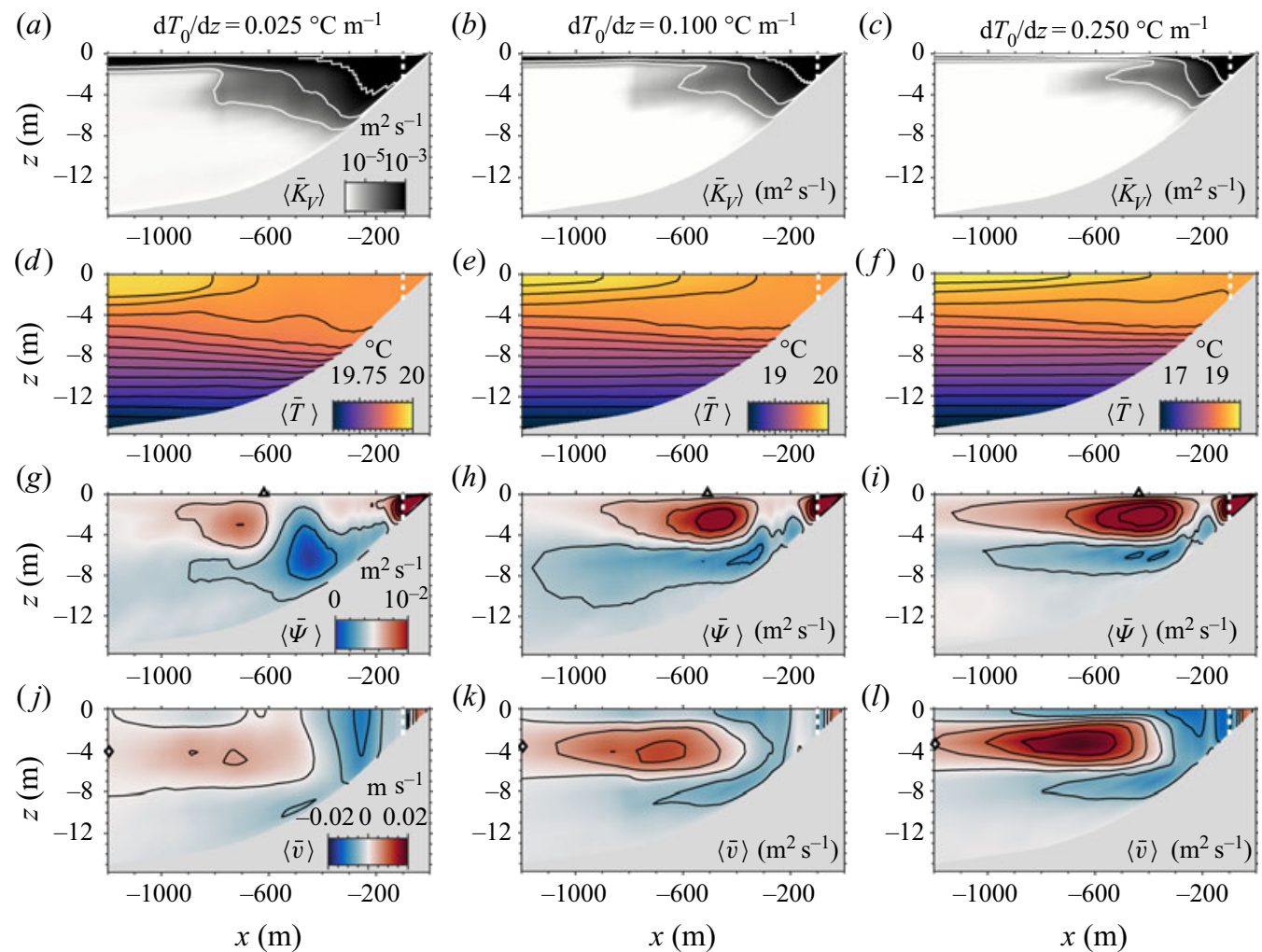

Figure 3. Mean $(x, z)$-sections of $(a)-(c)$ vertical eddy diffusivity $\left\langle\bar{K}_{V}\right\rangle$ on a $\log _{10}$ scale with white contours at $\left\{10^{-5}, 10^{-4}, 10^{-3}\right\} \mathrm{m}^{2} \mathrm{~s}^{-1},(d)-(f)$ temperature $\langle\bar{T}\rangle$ with black contour interval $\Delta z\left(\mathrm{~d} T_{0} / \mathrm{d} z\right)\left[{ }^{\circ} \mathrm{C}\right]$ with initial $\Delta z=1 \mathrm{~m}$ vertical separation, $(g)-(i)$ overturning streamfunction $\langle\bar{\Psi}\rangle$ contoured at $2 \times 10^{-3} \mathrm{~m}^{2} \mathrm{~s}^{-1}$, where red (blue) indicates clockwise (counter-clockwise) circulation and $(j)-(l)$ along-shore velocity $\langle\bar{v}\rangle$ with contour interval of $5 \times 10^{-3} \mathrm{~m} \mathrm{~s}^{-1}$. The left, centre and right columns represent initial stratifications $\mathrm{d} T_{0} / \mathrm{d} z=\{0.025,0.1,0.25\}^{\circ} \mathrm{C} \mathrm{m}^{-1}$, respectively. Note, the $T$ colour scale and contour interval vary from $(d)-(f)$. The diamond in $(j)-(l)$ indicates the $t=48 \mathrm{~h}$ sorted temperature anomaly $\Delta T_{s}^{48 \mathrm{~h}}$ zero-crossing depth $z=-d_{s}^{48 \mathrm{~h}}$, and the triangle in $(g)-(i)$ denotes the cross-shore location where $h(x)=3 d_{s}^{48 \mathrm{~h}}$. The $L_{S Z}=100 \mathrm{~m}$ wide surf zone is delimited with a vertical dashed white line.

The vertical thickness of this TRC-mixed fluid decreases with increased $\mathrm{d} T_{0} / \mathrm{d} z$ (figure $3 d-f$ ), due to a weaker $\left\langle\bar{K}_{V}\right\rangle$. Consequently, isotherm slopes near the base of the intrusion decrease by roughly half from $2 \times 10^{-3}$ with a factor of 10 increase in $\mathrm{d} T_{0} / \mathrm{d} z$. Reduced isotherm slopes also cause the cross-shore location of intrusion surface and bottom fronts, where well-mixed fluid intersects the surface and bottom, to move closer to shore with increasing $\mathrm{d} T_{0} / \mathrm{d} z$ (not shown).

Around $x=-500 \mathrm{~m}$, the baroclinic pressure is high onshore of upward sloping isotherms $(z \gtrsim-4 \mathrm{~m}$, figure $3 d-f)$, is maximum within the low-stratification intrusion $(z \approx-4 \mathrm{~m}$ ), then decreases and becomes low (relative to $x<-500$ ) below the intrusion $(z \lesssim-6 \mathrm{~m})$ before returning to near zero at depth. The induced baroclinic pressure gradient drives an $(x, z)$-overturning circulation, described by streamfunction $\langle\bar{\Psi}\rangle$, where cross-shore and vertical Lagrangian velocities (Eulerian + Stokes drift) are $\left(\bar{u}_{L}, \bar{w}_{L}\right)=$ $\left(-\partial_{z}, \partial_{x}\right) \bar{\Psi}$. Near the inner-shelf $\langle\bar{\Psi}\rangle$ maximum (triangles, top of figure $\left.3 g-i\right)$, the cross-shore Lagrangian $\bar{u}_{L}$ is comprised of onshore Stokes drift, with a surface maximum, 


\section{Self-similar inner-shelf response to transient rip currents}

and a mostly offshore Eulerian flow, with a sub-surface minimum at the depth of the intrusion, that are of similar magnitudes. The resulting mean inner-shelf $\langle\bar{\Psi}\rangle$ circulation is clockwise near surface and counter-clockwise near bottom (red and blue, respectively, figure $3 g-i$ ), leading to sub-surface offshore transport and near surface and bottom onshore transport for $x<-3 L_{S Z}$.

The circulation exchanges well-mixed fluid from the TRC region with stratified fluid from offshore. As stratification increases the near-surface clockwise cell moves onshore and intensifies, confining the counter-clockwise cell below mid-depth and slightly weakening. The location of inner-shelf $\langle\bar{\Psi}\rangle$ extrema, and associated maximum $\left\langle\bar{u}_{L}\right\rangle$, vary with $\mathrm{d} T_{0} / \mathrm{d} z$, generally occurring onshore of $x \approx-5 L_{S Z}$, loosely defined here as the near field with $x<-5 L_{S Z}$ the far field. The unstratified inner-shelf circulation is limited to the clockwise cell (not shown), which occupies the full water column and is consistent with onshore Stokes drift and offshore undertow-induced exchange (cf. figure $6 b$ in Kumar \& Feddersen 2017a). Scalings for the vertical thickness of the TRC-mixed fluid, the mean cross-shore velocity $\left\langle\bar{u}_{L}\right\rangle$ and near-field/far-field boundary are derived in $\S 5.1$.

Offshore of $x \approx-5 L_{S Z}$, a far-field intrusion forms with cross-shore sloping isotherms in approximate thermal wind balance with vertically sheared along-shore current $\langle\bar{v}\rangle$ (Kumar \& Feddersen 2017b). Note that the periodic $y$-boundary conditions do not permit a mean along-shore pressure gradient. The depth of the maximum northward (positive) $\langle\bar{v}\rangle$ decreases weakly with increasing $\mathrm{d} T_{0} / \mathrm{d} z$ (diamonds, figure $3 j-l$ ) and occurs roughly where isotherms switch from being upward to downward sloping toward shore $(z \approx-4 \mathrm{~m}$ in figures $3 d-f$ and $3 j-3 l)$. The maximum $\langle\bar{v}\rangle$ increases from $0.6 \times 10^{-2}$ to $1.8 \times 10^{-2} \mathrm{~m} \mathrm{~s}^{-1}$ for an increase in $\mathrm{d} T_{0} / \mathrm{d} z$ from $0.025^{\circ} \mathrm{C} \mathrm{m}^{-1}$ to $0.25^{\circ} \mathrm{C} \mathrm{m}^{-1}$ (figures $3 j$ and $3 l$ ). Maxima occur offshore of the inner-shelf $\langle\bar{\Psi}\rangle$ extrema. The offshore extent of the positive along-shore current increases with $\mathrm{d} T_{0} / \mathrm{d} z$, while the vertical thickness decreases weakly. In the TRC region $\left(x>-4 L_{S Z}\right)$ and offshore of the surf zone $\left(x<-L_{S Z}\right),\langle\bar{v}\rangle$ is negative with a weak vertical structure. In the surf zone, $\langle\bar{v}\rangle$ is much weaker, positive and depth uniform. Scalings for the far-field mean isotherm slope and along-shore velocity are derived in $\S 5.2$.

\subsection{Fluctuating inner-shelf response with varying stratification}

The effects of initial stratification $\mathrm{d} T_{0} / \mathrm{d} z$ on eddy variability are introduced using a 24-48 h time and quadratic along-shore average, e.g. for temperature,

$$
\text { r.m.s. }\left\{T^{\prime}\right\}=\left\langle\overline{T^{\prime 2}}\right\rangle^{1 / 2} \text {. }
$$

The along-shore-averaged temperature and circulation evolve over the 24-48 $\mathrm{h}$ averaging period, however, secular changes in offshore stratification and the mean position of intrusion fronts are small relative to the time and along-shore variability. Thus, (3.1) quantifies fluctuations $\left(T^{\prime}, u^{\prime}, w^{\prime}\right)$ primarily due to stochastic TRCs, with some contribution from internal gravity wave activity (e.g. Manins 1976). Two levels of stratification, $\mathrm{d} T_{0} / \mathrm{d} z=\{0.025,0.25\}^{\circ} \mathrm{C} \mathrm{m}^{-1}$, are shown in left and right columns of figure 4 , respectively. For both cases, the largest temperature fluctuations occur outside the surf zone $(x<-100 \mathrm{~m})$ and at the base of the TRC-mixed fluid $(\approx 6-8 \mathrm{~m}$ depth, figure $3 d-f)$, where isotherms are vertically compressed $\Delta z \leq 1 \mathrm{~m}$ (figure $3 d-f$ ). Note that the maximum is roughly $10 \times$ larger in figure $4(b)$ than figure $4(a)$, consistent with the $10 \times$ larger $\mathrm{d} T_{0} / \mathrm{d} z$ and indicating roughly a $1 \mathrm{~m}$ r.m.s. vertical isotherm displacement. The depth of sub-surface maxima decreases with increasing $\mathrm{d} T_{0} / \mathrm{d} z$. Vertical bands of slightly 


\section{D.J. Grimes and F. Feddersen}
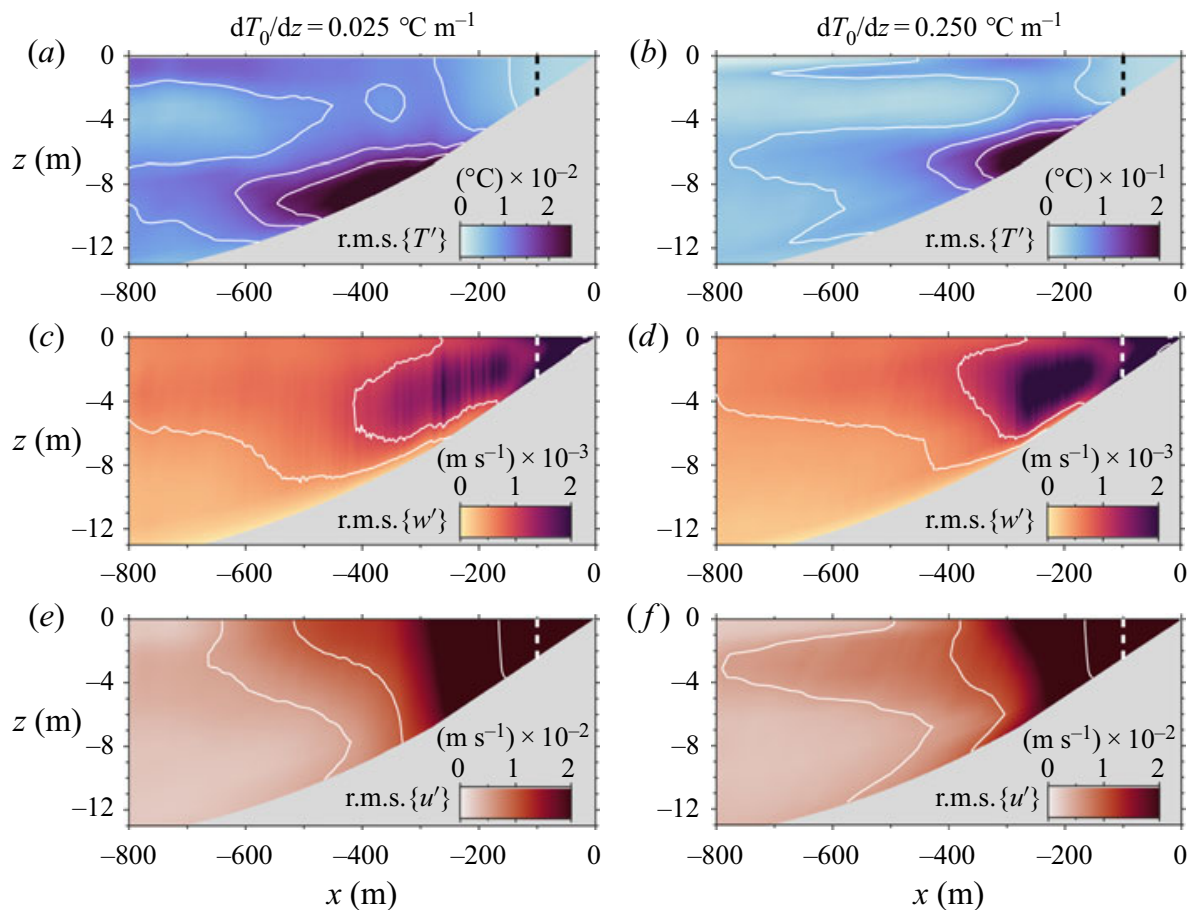

Figure 4. Time and along-shore root-mean-square $(3.1)(a, b)$ temperature perturbation r.m.s. $\left\{T^{\prime}\right\}(x, z)$ with white contour intervals of 0.005 and $0.05^{\circ} \mathrm{C}$ in $(a)$ and $(b)$, respectively; $(c, d)$ vertical velocity r.m.s. $\left\{w^{\prime}\right\}$ with white contours at $\{0.5,1\} \times 10^{-3} \mathrm{~m} \mathrm{~s}^{-1}$; and $(e, f)$ cross-shore velocity r.m.s. $\left\{u^{\prime}\right\}$ with contours at $\{0.5,1,5\} \times 10^{-2} \mathrm{~m} \mathrm{~s}^{-1}$. Panels $(a, c, e)$ have $\mathrm{d} T_{0} / \mathrm{d} z=0.025^{\circ} \mathrm{C} \mathrm{m}^{-1}$ and correspond to panels $(a, d, g, j)$ in figure 3; and panels $(b, d, f)$ have $\mathrm{d} T_{0} / \mathrm{d} z=0.25^{\circ} \mathrm{C} \mathrm{m}^{-1}$, corresponding to panels $(c, f, i, l)$ in figure 3 . Note the approximate factor of 10 change in colour scale between $(a)$ and $(b)$. The $L_{S Z}=100 \mathrm{~m}$ wide surf zone is delimited with a vertical dashed line, and the bottom is grey.

elevated r.m.s. $\left\{T^{\prime}\right\}$ above the maxima extend to the surface. Temperature variability also increases near surface in association with a surface temperature front (e.g. $-4 L_{S Z}<x<$ $-2 L_{S Z}$, figure $\left.2 b\right)$.

Outside the surf zone, $x<-L_{S Z}$, the largest vertical velocity fluctuations r.m.s. $\left\{w^{\prime}\right\} \approx$ $2 \times 10^{-3} \mathrm{~m} \mathrm{~s}^{-1}$ occur mid-water column and predominately onshore of $x=-4 L_{S Z}$, consistent with Kumar \& Feddersen (2017b). The vertical velocities heave isotherms (e.g. figure $2 e$ ) and are responsible for the vertical band of r.m.s. $\left\{T^{\prime}\right\}$ in figure $4(a, b)$. Vertical isotherm displacement combined with strong horizontal advection generate temperature inversions and elevated $K_{V}$. The magnitude of vertical eddy velocities increases slightly with $\mathrm{d} T_{0} / \mathrm{d} z$, while the cross-shore extent decreases. However, the change is minor when contrasted with the increase in temperature fluctuations, suggesting TRC-induced vertical velocity fluctuations are only weakly affected by stratification.

The cross-shore directed eddy velocity peak r.m.s. $\left\{u^{\prime}\right\} \approx 0.22 \mathrm{~m} \mathrm{~s}^{-1}$ occurs within the surf zone, $x>-L_{S Z}$, and decreases to $<5 \times 10^{-2} \mathrm{~m} \mathrm{~s}^{-1}$ by $x=-2 L_{S Z}$ (figure $4 e$ ). For $x>-2 L_{S Z}$, r.m.s. $\left\{u^{\prime}\right\}$ the vertical structure is weak and largely independent of $\mathrm{d} T_{0} / \mathrm{d} z$. Vertical structure increases over $-3 L_{S Z}<x<-2 L_{S Z}$, particularly for cases with higher stratification (e.g. figure $4 f$ ). Offshore of $-4 L_{S Z}$, r.m.s. $\left\{u^{\prime}\right\}$ continues to decrease and tends toward a sub-surface maximum roughly following $\langle\bar{\Psi}\rangle$ streamlines, suggesting offshore advection of the residual eddy velocities. The weak variation in eddy velocity statistics 


\section{Self-similar inner-shelf response to transient rip currents}

with $\mathrm{d} T_{0} / \mathrm{d} z$ compared to the temperature fluctuations increasing in proportion to $\mathrm{d} T_{0} / \mathrm{d} z$ suggests TRC-induced overturns may have larger associated diapycnal mixing.

\section{Results: effect of stratification and TRCs on the energetics}

To develop scalings for the inner-shelf mean circulation, i.e. $\langle\bar{\Psi}\rangle$ and $\langle\bar{v}\rangle$, we examine the energetic response to surf-zone and TRC-induced mixing with varying initial stratification. This analysis identifies an energetics based vertical length scale that quantifies the domain potential energy response. The energetics length scale is used to derive inner-shelf mean stratification and circulation scalings in subsequent sections. First, the domain-integrated kinetic and potential energy evolution are presented for $\mathrm{d} T_{0} / \mathrm{d} z=0.25^{\circ} \mathrm{C} \mathrm{m}^{-1}$, followed by an analysis of the spatial structure and temporal evolution with varying stratification. The kinetic energy is decomposed using (2.3) into contributions from specific eddy kinetic energy,

$$
\mathcal{E}_{k^{\prime}}(x, z, t)=\frac{\varrho}{2} \overline{\left(u^{\prime 2}+v^{\prime 2}\right)},
$$

where the neglected vertical velocities are an order of magnitude smaller (figure $4 b, c$ ). Specific $y$-mean kinetic energy $\mathcal{E}_{\bar{k}}$ is defined similar to (4.1) using $\left(\bar{u}_{L}, \bar{v}\right)$. As surf-zone breaking wave rotational forcing is the only external driver of eddy variability (e.g. Grimes et al. 2020b), $\mathcal{E}_{k^{\prime}}$ quantifies energy in the TRC-induced eddy field. The domain $(x, z)$-area $A$-integrated $\mathcal{E}_{k^{\prime}}$ is,

$$
E_{k^{\prime}}^{(x, z)}(t)=\int_{A} \mathcal{E}_{k^{\prime}} \mathrm{d} A,
$$

where superscripts indicate the integrated dimensions. The domain-integrated mean kinetic energy $E_{\bar{k}}^{(x, z)}(t)$ is defined similar to $E_{k^{\prime}}^{(x, z)}$ (4.2) by substituting $\mathcal{E}_{\bar{k}}$ for $\mathcal{E}_{k^{\prime}}$. For stratified conditions, irreversible vertical mixing induces changes in along-shore mean specific potential energy relative to the initial condition $\rho_{0}(2.2)$, defined as (e.g. Kumar \& Feddersen 2017b),

$$
\Delta \mathcal{E}_{p}(x, z, t)=g z\left[\bar{\rho}(x, z, t)-\rho_{0}(z)\right] .
$$

For a closed domain, vertical mixing increases the domain area integrated potential energy, denoted $E_{p}^{(x, z)}(t)$ (the $\Delta$-prefix is dropped for domain-integrated quantities).

For $\mathrm{d} T_{0} / \mathrm{d} z=0.25^{\circ} \mathrm{C} \mathrm{m}^{-1}$, the TRC-induced eddy field reaches $85 \%$ of the $24-48 \mathrm{~h}$ time average $\left\langle E_{k^{\prime}}^{(x, z)}\right\rangle=6.24 \mathrm{~kJ} \mathrm{~m}^{-1}$ in roughly $1 \mathrm{~h}$ (black, figure 5). Subsequently, $E_{k^{\prime}}^{(x, z)}$ varies $\pm 15 \%$ of $\left\langle E_{k^{\prime}}^{(x, z)}\right\rangle$ over time scales of $\lesssim 3 \mathrm{~h}$ with no discernible linear trend for $t>1 \mathrm{~h}$, indicating the eddy field has equilibrated. The domain-integrated mean kinetic energy, $E_{\bar{k}}^{(x, z)}(t)$, increases continuously throughout the $48 \mathrm{~h}$ simulation but is much weaker than $E_{k^{\prime}}^{(x, z)}$, only reaching $\approx 0.75 \mathrm{~kJ} \mathrm{~m}^{-1}$ in $48 \mathrm{~h}$ (red, figure 5). The far-field along-shore flow $\bar{v}$ dominates $E_{\bar{k}}^{(x, z)}$ ( $\bar{u}_{L}$ contributions are minor); and $E_{\bar{k}}^{(x, z)}$ temporal variability is also relatively large, $\approx 25 \%$ of the $24-48 \mathrm{~h}$ mean (not shown). The domain-integrated potential energy change $E_{p}^{(x, z)}(t)$ also increases continuously, becoming larger than $E_{\bar{k}}^{(x, z)}$ after $\approx 30 \mathrm{~min}$ and larger than $\left\langle E_{k^{\prime}}^{(x, z)}\right\rangle$ after $22 \mathrm{~h}$ (blue, figure 5). The $E_{p}^{(x, z)}$ slope decreases slowly between $0<t \leq 12 \mathrm{~h}$, becoming approximately constant after $24 \mathrm{~h}$, suggesting the near-field exchange flow and TRC-region conversion have reached quasi-equilibrium. Continuous $E_{p}^{(x, z)}$ growth indicates continuous water-mass transformation. 


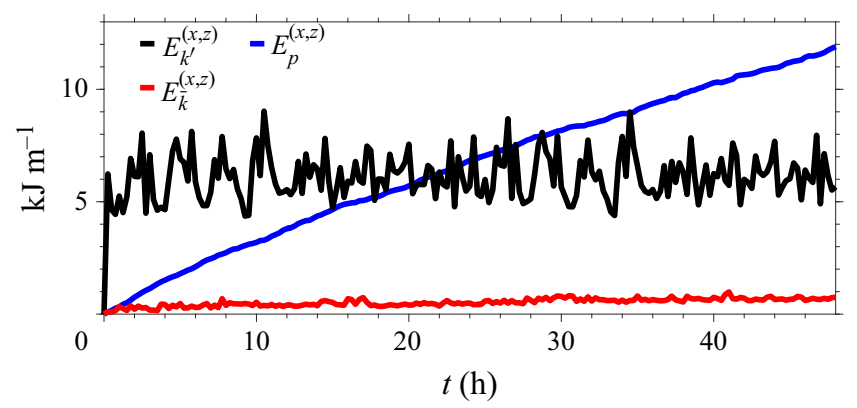

Figure 5. Time evolution of the $\mathrm{d} T_{0} / \mathrm{d} z=0.25^{\circ} \mathrm{C} \mathrm{m}^{-1}$ domain-integrated eddy kinetic energy $E_{k^{\prime}}^{(x, z)}(4.2)$ in black, mean kinetic energy $E_{\bar{k}}^{(x, z)}$ (red) and potential energy $E_{p}^{(x, z)}$ (blue).

\subsection{Eddy kinetic energy}

The vertical and cross-shore contraction of elevated mean tracer eddy diffusivity (figure $3 a-c$ ) and height of low-stratification fluid (figure $3 d-f$ ) with increasing $\mathrm{d} T_{0} / \mathrm{d} z$ can result from inner-shelf stratification limiting the offshore extent of TRC-induced velocity fluctuations $\left(u^{\prime}, v^{\prime}\right)$ or through suppression in the vertical scales of mixing. The specific eddy kinetic energy $\mathcal{E}_{k^{\prime}}$ (4.1) is analysed to determine whether TRC eddy velocities are affected by inner-shelf stratification. First, the cross-shore distribution of TRC energy with varying stratification is evaluated by depth integrating the time-averaged $\mathcal{E}_{k^{\prime}}$,

$$
E_{k^{\prime}}^{(z, t)}(x)=\int_{-h}^{\langle\bar{\eta}\rangle}\left\langle\mathcal{E}_{k^{\prime}}\right\rangle \mathrm{d} z
$$

where the superscripted $t$ indicates time-averaged integrand. The cross-shore varying $E_{k^{\prime}}^{(z, t)}$ is maximum in the surf zone and decreases rapidly offshore (figure $6 a$ ). At $x=-L_{S Z}$, $E_{k^{\prime}}^{(z, t)} \approx 25 \mathrm{~J} \mathrm{~m}^{-2}$ implies a depth-averaged velocity magnitude of $0.14 \mathrm{~m} \mathrm{~s}^{-1}$, consistent with the r.m.s. $\left\{u^{\prime}\right\}$ magnitude (figure $4 c$ ). For all simulations, $E_{k^{\prime}}^{(z, t)}$ consistently decreases offshore with no obvious $\mathrm{d} T_{0} / \mathrm{d} z$ dependence out to $x=-6 L_{S Z}$ (coloured lines, figure $6 a$ ). The $\mathrm{d} T_{0} / \mathrm{d} z=0$ case (black) differs for $x<-6 L_{S Z}$ as unstratified circulation response is fundamentally different than stratified simulations. The cross-shore $E_{k^{\prime}}^{(z, t)}$ structure suggests that the energetic TRC-induced eddies are confined within a few surf-zone widths from shore (Suanda \& Feddersen 2015).

The time-dependent $E_{k^{\prime}}^{(x, z)}(4.2)$ is also similar for all $\mathrm{d} T_{0} / \mathrm{d} z$ simulations (figure $6 b$ ), suggesting that TRC-induced eddy kinetic energy is dependent on the stochastic surf-zone rotational forcing, which is the same for all simulations. The absence of $E_{k^{\prime}}^{(x, z)}$ secular growth indicates that surf-zone eddy kinetic energy generation is in equilibrium with its domain-integrated destruction through conversion (to potential and along-shore mean kinetic energy) and viscous dissipation. The similar $E_{k^{\prime}}^{(z, t)}$ and $E_{k^{\prime}}^{(x, z)}$ for all $\mathrm{d} T_{0} / \mathrm{d} z>0$ indicate that TRC energetics are unaffected by stratification.

The cross-shore kinetic energy flux $\left(F_{k^{\prime}}\right)$, quantified as,

$$
F_{k^{\prime}}=\int_{-h}^{\langle\bar{\eta}\rangle}\left\langle\overline{u_{L} \mathcal{E}_{k^{\prime}}}\right\rangle \mathrm{d} z,
$$

is used to determine where on the inner-shelf the bulk of TRC energy is dissipated. Eddy transport dominates $F_{k^{\prime}}$, i.e. from $u^{\prime}\left(u^{\prime 2}+v^{\prime 2}\right)$, and negative $F_{k^{\prime}}$ indicates a 

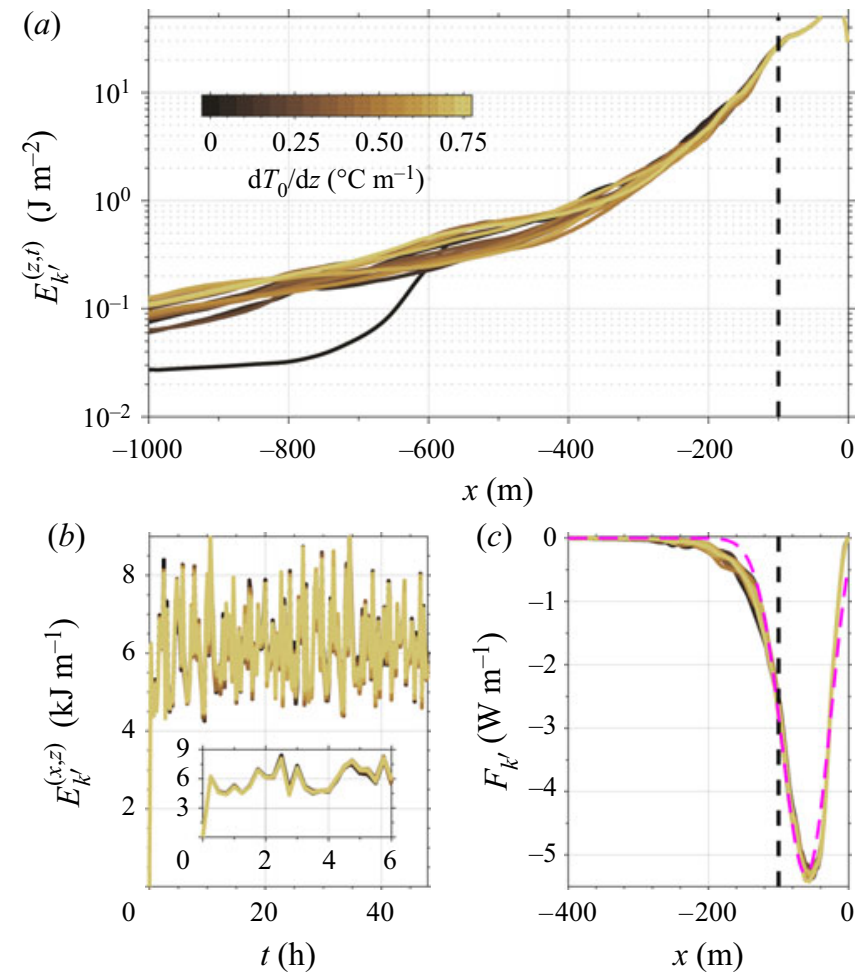

Figure 6. (a) Cross-shore distribution of depth-integrated mean eddy kinetic energy $E_{k^{\prime}}^{(z, t)}$ at varying $\mathrm{d} T_{0} / \mathrm{d} z$ (colours). (b) The $48 \mathrm{~h}$ time evolution of along-shore-averaged depth and cross-shore integrated eddy kinetic energy $E_{k^{\prime}}^{(x, z)}$, with inset showing $6 \mathrm{~h}$ spin-up period. (c) Distribution of cross-shore directed kinetic energy flux $F_{k^{\prime}}(4.5)$, with Gaussian barotropic rotational velocity scaling (6.5)-(6.9) in magenta. In $(a, c)$, the vertical dashed line indicates $x=-L_{S Z}$.

surf-zone to inner-shelf flux (figure $6 c$ ). The $F_{k^{\prime}}$ is similar for all $\mathrm{d} T_{0} / \mathrm{d} z$, confirming that TRCs are largely unaffected by initial stratification, and consistent with r.m.s. $\left\{u^{\prime}\right\}$ and r.m.s. $\left\{w^{\prime}\right\}$ (figure $4 b, c$ ). As eddy kinetic energy statistics are approximately stationary for $t>1 \mathrm{~h}$, the $x<-L_{S Z}$ slope of $F_{k^{\prime}}$ indicates either frictional damping of $E_{k^{\prime}}^{(z, t)}$ or conversion to potential energy. Rapid $F_{k^{\prime}}$ divergence over $-4 L_{S Z} \leq x \leq-1 L_{S Z}$ for all $\mathrm{d} T_{0} / \mathrm{d} z$, indicates that dissipation/conversion occurs over a similar cross-shore region independent of stratification. The magnitude and cross-shore $F_{k^{\prime}}$ distribution is consistent with previously derived TRC velocity scaling by Suanda \& Feddersen (2015) (magenta, figure 6(c); discussed in detail in $\S 6.3$ ).

\subsection{Background and available potential energy}

The TRC-induced mixing over $x>-4 L_{S Z}$ increases $E_{p}^{(x, z)}$ (e.g. figure 5) and drives inner-shelf mean circulation (figure 3 ). Here, the domain-integrated potential energy is evaluated to determine what fraction of TRC eddy kinetic energy is converted to potential energy. First, the along-shore-averaged specific potential energy (4.3) is decomposed into changes in background potential energy $\left(\Delta \mathcal{E}_{b}\right)$, a measure of irreversible mixing, and available $\left(\mathcal{E}_{a}\right)$ potential energy, a measure of reversible buoyancy fluxes that drive mean 


\section{D.J. Grimes and F. Feddersen}

circulation (e.g. Winters et al. 1995; MacCready \& Giddings 2016),

$$
\Delta \mathcal{E}_{p}(x, z, t) \equiv \Delta \mathcal{E}_{b}(z, t)+\mathcal{E}_{a}(x, z, t) .
$$

The change in background potential energy is defined as,

$$
\Delta \mathcal{E}_{b}(z, t)=g z\left[\rho_{s}(z, t)-\rho_{0}(z)\right],
$$

in terms of the sorted density profile $\rho_{s}(z, t)$, derived from $\bar{\rho}(x, z, t)$ by adiabatically redistributing water parcels such that the sorted isopycnals are horizontal, thus giving the minimum potential energy state of the system (Winters et al. 1995). Irreversible mixing modifies $\rho_{s}$ and increases the background potential energy. TRC-region $(x>$ $\left.-4 L_{S Z}\right)$ mixing also modifies the local specific potential energy, manifested as sloped $\langle\bar{T}\rangle$ isotherms (e.g. figure $3 d-f$ ). Specific available potential energy $\left(\mathcal{E}_{a}\right)$ is commonly defined as the work required to vertically displace parcels through the sorted density field, as determined by $\rho_{s}$ (e.g. Winters \& Barkan 2013; MacCready \& Giddings 2016),

$$
\mathcal{E}_{a}(x, z, t)=\int_{\bar{z}_{s}}^{z} g\left[\bar{\rho}(x, z, t)-\rho_{s}\left(z^{\prime}, t\right)\right] \mathrm{d} z^{\prime},
$$

where $\bar{z}_{s}$ is the $z$-level corresponding to $\rho_{s}\left(\bar{z}_{s}\right)=\bar{\rho}(x, z, t)$. Thus, $\mathcal{E}_{a}(4.8)$ is a measure of the available potential energy due to cross-shore sloping along-shore averaged isopycnals, and does not include contributions from along-shore varying TRC-induced eddies whose statistics are largely stationary (cf. § 4.1).

\subsubsection{Domain-integrated background and available potential energy evolution with varying stratification}

The temporal evolution of potential energy is analysed using the domain $(x, z)$-areaintegrated background potential energy change,

$$
E_{b}^{(x, z)}(t)=\int_{-\max \{h\}}^{0} \Delta \mathcal{E}_{b}(z, t) \mathcal{L}_{x}(z) \mathrm{d} z,
$$

where the area increment $\left(\mathcal{L}_{x}(z) \mathrm{d} z\right)$ accounts for the sloping bottom, such that vertically integrating $\left(\mathcal{L}_{x}(z) \mathrm{d} z\right)$ gives the domain $(x, z)$-area $A$. The $(x, z)$-area integrated available potential energy is given by the residual,

$$
E_{a}^{(x, z)}(t) \equiv E_{p}^{(x, z)}-E_{b}^{(x, z)}
$$

Initially, at $t=0, E_{b}^{(x, z)}$ and $E_{a}^{(x, z)}$ are both zero (figure 7). For stratified cases, both quantities subsequently increase as irreversible mixing and reversible buoyancy fluxes modify the density field. Thus, the potential energy growth rate $\left(\partial_{t} E_{p}^{(x, z)}\right)$ is a measure of the conversion from kinetic to potential energy. For $t>24 \mathrm{~h}$, the growth rate $\partial_{t} E_{b}^{(x, z)}$ is roughly constant, indicating that once the near-field stratification and circulation equilibrate, the water-mass transformation also becomes quasi-steady. The $24-48 \mathrm{~h}$ mean growth rate increases with increasing $\mathrm{d} T_{0} / \mathrm{d} z$ (figure $7 a$ ), from 0.006 to $0.13 \mathrm{~W} \mathrm{~m}^{-1}$ for $\mathrm{d} T_{0} / \mathrm{d} z=0.0125^{\circ} \mathrm{C} \mathrm{m}^{-1}$ and $0.75^{\circ} \mathrm{C} \mathrm{m}^{-1}$, respectively. Similarly, $E_{a}^{(x, z)}$ increases with $\mathrm{d} T_{0} / \mathrm{d} z$, but is smaller than $E_{b}^{(x, z)}$ by roughly an order of magnitude (figure $7 b$ ). Thus, only a small fraction, $O(10 \%)$, of the potential energy changes are available for driving inner-shelf circulation. The $E_{a}^{(x, z)}$ temporal variability increases for strong stratification. 


\section{Self-similar inner-shelf response to transient rip currents}
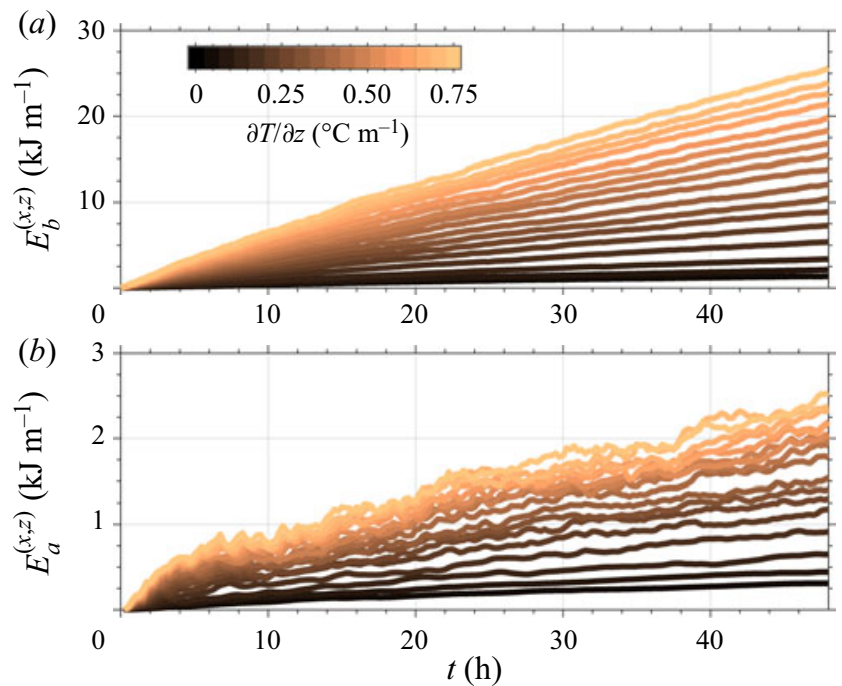

Figure 7. Time evolution of domain-integrated (a) background potential energy $E_{b}^{(x, z)}(4.9)$ and $(b)$ available potential energy $E_{a}^{(x, z)}$ (4.10) at varying levels of initial stratification $\mathrm{d} T_{0} / \mathrm{d} z$ (colours). Note the factor of 10 difference in ordinate scale between $(a)$ and $(b)$.

For vertical mixing localized to $-4 L_{S Z}<x<-L_{S Z}$, modelled $E_{p}^{(x, z)}$ growth rates imply a mean vertical buoyancy flux varying between $0.3 \times 10^{-4}$ and $5 \times 10^{-4} \mathrm{~W} \mathrm{~m}^{-2}$, similar to inner-shelf observations of individual internal bore mixing events (Sinnett et al. 2018).

The increasing mean growth rate $\left\langle\partial_{t} E_{p}^{(x, z)}\right\rangle$ with initial stratification suggests the fraction of surf-zone-generated $E_{k^{\prime}}^{(x, z)}$ converted into $E_{p}^{(x, z)}$ increases with $\mathrm{d} T_{0} / \mathrm{d} z$. This implies the domain-integrated frictional dissipation rate is reduced with $\mathrm{d} T_{0} / \mathrm{d} z$ for energy conservation (not shown). A domain average or bulk conversion efficiency $\gamma_{E}$ is estimated as,

$$
\gamma_{E}=\frac{\left\langle\partial_{t} E_{p}^{(x, z)}\right\rangle}{F_{k^{\prime}}^{(s z)}},
$$

where the eddy kinetic energy flux at $x=-L_{S Z}, F_{k^{\prime}}^{(s z)} \equiv F_{k^{\prime}}\left(x=-L_{S Z}\right)$, is used for the source rate. As initial stratification increases, $\gamma_{E}$ increases from $\approx 0.003$ to 0.05 (circles, figure 8). Thus, only a small fraction of $F_{k^{\prime}}^{(s z)}$ goes to increasing $E_{p}^{(x, z)}$. As the mean cross-shore kinetic energy flux $F_{k^{\prime}}$ is stationary and not affected by initial stratification, the change in $\gamma_{E}$ with $\mathrm{d} T_{0} / \mathrm{d} z$ is predominately due to changes in $E_{p}^{(x, z)}$ growth rate. As $E_{p}^{(x, z)}$ is dominated by $E_{b}^{(x, z)}, \gamma_{E}$ is an approximate measure of the domain-averaged mixing efficiency (Gregg et al. 2018). Increasing $\gamma_{E}$ also indicates the surf zone generates more than sufficient energy to mix the stratified inner shelf. For $\mathrm{d} T_{0} / \mathrm{d} z \geq 0.1^{\circ} \mathrm{C} \mathrm{m}^{-1}$, the model $\gamma_{E}$ is roughly consistent with the cross-shore advective buoyancy flux scaling (5.16; crosses, figure 8) derived in $\$ 5.3$ using a potential energy based length scale and baroclinic cross-shore velocity scale.

\subsubsection{Evolution of sorted density $\rho_{\text {s }}$ profile}

Domain-integrated $E_{b}^{(x, z)}$ increases more rapidly for larger $\mathrm{d} T_{0} / \mathrm{d} z$ (figure $7 a$ and 8 ), indicating an increased diapycnal mixing modification of the domain density distribution, 


\section{D.J. Grimes and F. Feddersen}

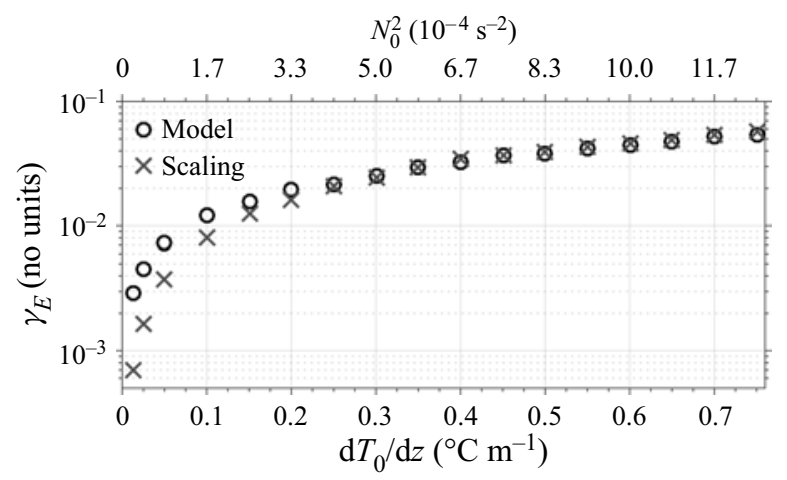

Figure 8. Domain-integrated and 24-48 h averaged $E_{k^{\prime}}^{(x, z)}$ to $E_{p}^{(x, z)}$ bulk conversion efficiency $\gamma_{E}$ (4.11, circles) vs $\mathrm{d} T_{0} / \mathrm{d} z$ (bottom) and $N_{0}^{2}$ (top). Also shown is a near-field advective buoyancy flux scaling (5.16; crosses) based on sorted temperature anomaly zero-crossing depth $d_{s}$ and cross-shore baroclinic velocity scale $\mathcal{U}(5.2)$.
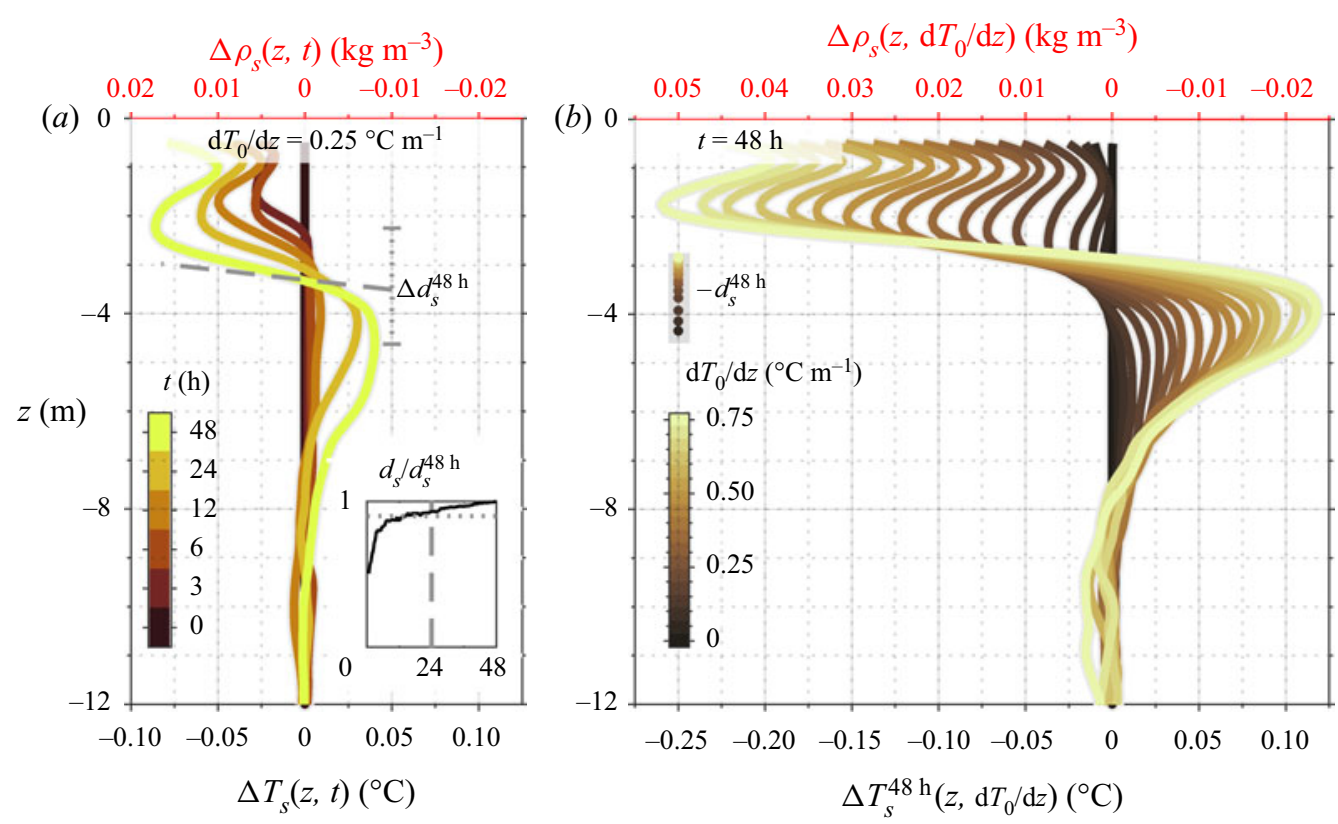

Figure 9. Temperature anomaly $\Delta T_{s}=T_{s}(z, t)-T_{0}(z)$ of the sorted state $T_{s}$ relative to the initial temperature profile for $(a)$ run initialized with $\mathrm{d} T_{0} / \mathrm{d} z=0.25^{\circ} \mathrm{C} \mathrm{m}^{-1}$ at several times (colours) showing the effect of irreversible mixing on the background state, and $(b)$ at model time $t=48 \mathrm{~h}$ for varying $\mathrm{d} T_{0} / \mathrm{d} z$ (colours). In $(a)$ the dashed grey line indicates minimum slope $\partial_{z} \Delta T_{s}=-\mathrm{d} T_{0} / \mathrm{d} z$, with intercept at the $t=48 \mathrm{~h}$ zero-crossing depth $z=-d_{s}^{48 \mathrm{~h}}$; the $t=48 \mathrm{~h}$ vertical extremum separation $\Delta T_{s}^{48 \mathrm{~h}}$ is also shown; the inset shows the time evolution of $d_{s} / d_{s}^{48 \mathrm{~h}}$ (black), with the start of the averaging period $t=24 \mathrm{~h}$ and the $90 \%$ of $d_{s}^{48 \mathrm{~h}}$ locations in grey. In $(b)$ the $t=48 \mathrm{~h}$ zero-crossing depths $d_{s}^{48 \mathrm{~h}}$ are shown for each $\mathrm{d} T_{0} / \mathrm{d} z$. Note, in the top axes of $(a, b)$ $\Delta \rho_{s}$ increases right to left.

and thus the structure of sorted density profile $\rho_{s}(z, t)$ (4.7). As available potential energy is a source of inner-shelf mean circulation (4.8) and is also determined via $\rho_{s}(z, t)$, the vertical structure of $\rho_{s}$ provides insight into how TRC mixing modifies the domain density distribution. Here, the effect of $N_{0}$ on the sorted density anomaly $\Delta \rho_{s}(z, t)=$ 
$\rho_{s}(z, t)-\rho_{0}(z)$ evolution (or sorted temperature anomaly $\Delta T_{s}=-\Delta \rho_{s} /(\varrho \alpha)$ ), relative to the initial condition $\rho_{0}(z)$ and $T_{0}(z)$, is examined (top and bottom axes, figure 9).

As surf zone and TRC mixing modify the inner-shelf temperature, a positive near-surface density anomaly $\Delta \rho_{s}$ develops (density increases right to left in figure $9 a$ ), or negative $\Delta T_{s}$, indicating dense (colder) fluid raised to a higher $z$-level. A corresponding sub-surface $\Delta \rho_{s}<0\left(\Delta T_{s}>0\right)$ also grows, indicating less dense (warmer) water mixed to a lower $z$-level. The vertical location where $\Delta T_{s}=0$ is defined as the zero-crossing depth $z=-d_{s}$, and indicates the vertical location where the average temperature of fluid mixed in the TRC region intersects the initial condition $T_{0}(z)$. From $3 \leq t \leq 48$ $\mathrm{h}$, the $\mathrm{d} T_{0} / \mathrm{d} z=0.25^{\circ} \mathrm{C} \mathrm{m}^{-1}$ zero-crossing depth $d_{s}$ increases from $\approx 2.5$ to $3.3 \mathrm{~m}$ indicating both continuous water-mass transformation and decreasing average temperature of TRC-mixed fluid. Initially $(t<3 \mathrm{~h}), d_{s}$ increases rapidly and becomes quasi-steady after $\approx 12 \mathrm{~h}$, increasing by $\lesssim 10 \%$ over $24-48 \mathrm{~h}$ (inset, figure $9 a$ ), consistent with a quasi-constant $\partial_{t} E_{b}^{(x, z)}$ for $t>24 \mathrm{~h}$ (figure $7 a$ ). At $t=48 \mathrm{~h}$, appreciable $\Delta \rho_{s}$ and $\Delta T_{s}$ are limited to approximately $z>-3 d_{s}$, or $z>-10 \mathrm{~m}$ for $\mathrm{d} T_{0} / \mathrm{d} z=0.25^{\circ} \mathrm{C} \mathrm{m}^{-1}$ (figure $9 a$ ). The time equilibration of $d_{s}$ is similar for all $\mathrm{d} T_{0} / \mathrm{d} z>0$, and is consistent with previous localized mixing experiments, where the mixed layer depth stabilizes when the gravity-driven export of mixed fluid away from the mixing region balances the entrainment of stratified fluid from below (e.g. Maxworthy \& Monismith 1988).

Conceptually, TRC-enhanced vertical mixing decreases the $z=-d_{s}$ sorted density/ temperature stratification, i.e. $\partial T_{S} / \partial z$, relative to the initial stratification $\mathrm{d} T_{0} / \mathrm{d} z$. The sorted stratification reduction occurs near $d_{s}$ because baroclinic circulation $\bar{\Psi}$ exports mixed fluid with characteristic $T \approx T_{0}\left(-d_{s}\right)$ from the TRC region, thereby increasing the $(x, z)$-area of this mixed water mass. The increasing area of $T_{0}\left(-d_{s}\right)$ fluid comes at the expense of the area between isotherms of the stratified fluid from which it was formed. The reduced area between these isotherms manifests as decreased vertical separation, such that, $\partial T_{S} / \partial z>\mathrm{d} T_{0} / \mathrm{d} z$ above and below the $\Delta T_{s}$ extrema, with vertical separation $\Delta d_{S}$ (right, figure $9 a$ ). Continuous water-mass transformation causes the magnitude of $\Delta T_{S}$ extrema to increase with time (figure $9 a$ ). Since $T_{s}$ is positive, definite the minimum possible slope $\partial T_{s} / \partial z=0$ implies a minimum $\partial_{z} \Delta T_{s}=-\mathrm{d} T_{0} / \mathrm{d} z$ (dashed grey line, figure $9 a$ ). Thus, the range of $\Delta T_{s}$ extrema is constrained by $\max \left\{\Delta T_{s}\right\}-\min \left\{\Delta T_{s}\right\} \leq\left(\Delta d_{s} \mathrm{~d} T_{0} / \mathrm{d} z\right)$.

The accumulated effect of TRC-enhanced mixing on $\Delta \mathcal{E}_{b}$ with varying initial stratification is evaluated using $t=48 \mathrm{~h}$ profiles of sorted temperature anomaly, denoted $\Delta T_{s}^{48 \mathrm{~h}}$ (figure $9 b$ ). The $t=48 \mathrm{~h}$ zero crossing, denoted $z=-d_{s}^{48 \mathrm{~h}}$, shoals from $z \approx-4.4$ to $\approx-2.9 \mathrm{~m}$ with increasing $\mathrm{d} T_{0} / \mathrm{d} z$ (coloured dots in figure $9 b$ ). Similar to $\mathrm{d} T_{0} / \mathrm{d} z=$ $0.25^{\circ} \mathrm{C} \mathrm{m}^{-1}$ (figure $9 a$ ), the largest $\Delta T_{s}^{48 \mathrm{~h}}$ are limited to approximately $z>-3 d_{s}^{48 \mathrm{~h}}$ for all $\mathrm{d} T_{0} / \mathrm{d} z\left(>12 \mathrm{~m}\right.$ for weak $\mathrm{d} T_{0} / \mathrm{d} z$ and $>8 \mathrm{~m}$ for strong $\left.\mathrm{d} T_{0} / \mathrm{d} z\right)$. The minimum slope $\partial_{z} \Delta T_{s}^{48 \mathrm{~h}} \approx-0.5 \mathrm{~d} T_{0} / \mathrm{d} z$ for all $\mathrm{d} T_{0} / \mathrm{d} z$ is half the minimum possible slope $-\mathrm{d} T_{0} / \mathrm{d} z$, suggesting that, on average, the surf-zone and TRC-mixed fluid has reduced stratification $\sim 0.5 \mathrm{~d} T_{0} / \mathrm{d} z$. The minimum $\partial_{z} \Delta T_{s}^{48 \mathrm{~h}}$ occurs slightly above $z=-d_{s}^{48 \mathrm{~h}}$, i.e. closer to the $\Delta T_{s}^{48 \mathrm{~h}}$ minima (not shown), causing asymmetry of $\Delta T_{S}^{48 \mathrm{~h}}$ extrema. The asymmetry is potentially due to differences in onshore transport between upper and lower $\langle\bar{\Psi}\rangle$ cells (cf. figure $3 g-i)$.

\subsubsection{Specific and depth-integrated available potential energy}

Localized TRC-region vertical mixing broadens isotherms toward shore (e.g. figure $3 d-f$ ), and strengthening inner-shelf circulation $(\langle\bar{\Psi}\rangle$ and $\langle\bar{v}\rangle)$ with $\mathrm{d} T_{0} / \mathrm{d} z$ indicates an enhanced baroclinic pressure gradient and specific available potential energy $\left(\mathcal{E}_{a}, 4.8\right)$, which 


\section{D.J. Grimes and F. Feddersen}
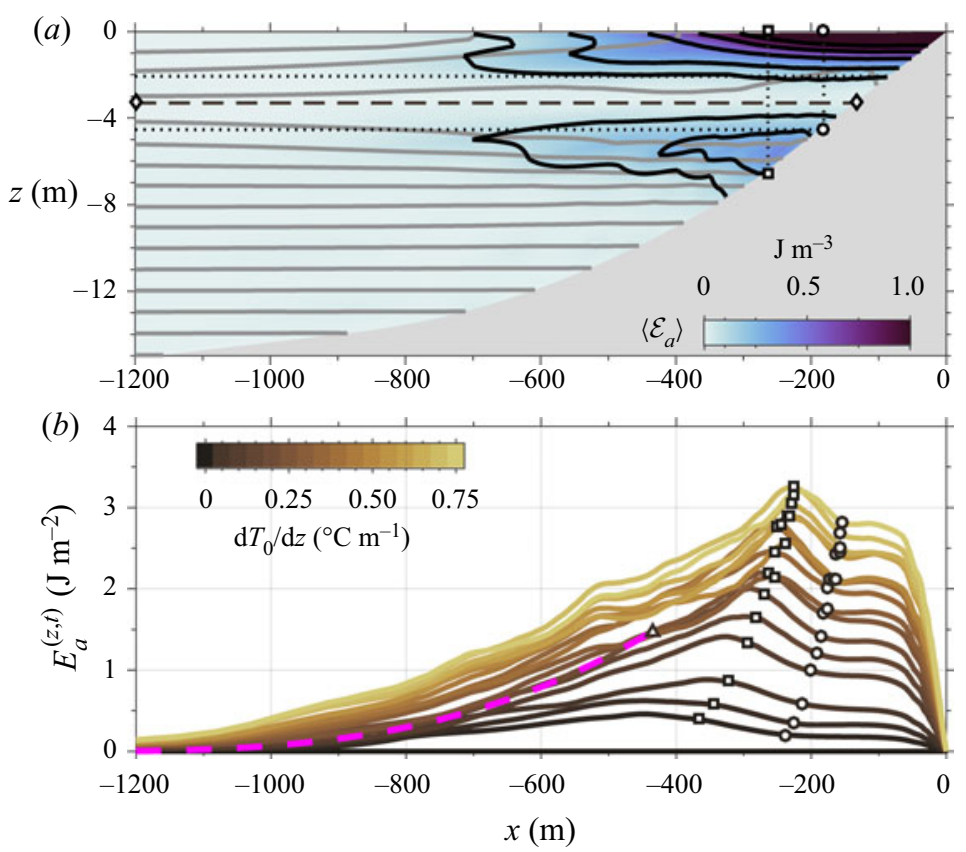

Figure 10. (a) Mean available potential energy $\left\langle\mathcal{E}_{a}\right\rangle(x, z)$ as a function of cross-shore and vertical directions. Black contours are $\{0.1,0.25,0.5,0.75,1\} \mathrm{J} \mathrm{m}^{-3}$, and grey contours are from corresponding $\langle\bar{T}\rangle$ at $0.25^{\circ} \mathrm{C}$ intervals (cf. figure $3 f$ ). (b) Depth-integrated $E_{a}^{(z, t)}$ vs cross-shore direction and $\mathrm{d} T_{0} / \mathrm{d} z$ (colours). In $(a)$ the $t=48 \mathrm{~h}$ zero-crossing depth $z=-d_{s}^{48} \mathrm{~h}$ is indicated by diamonds and a horizontal dashed line, and the approximate depths of $\Delta T_{s}$ extrema at $z=-\left(d_{s}^{48 \mathrm{~h}} \pm \Delta d_{s}^{48 \mathrm{~h}} / 2\right)$ are indicated by horizontal dotted lines. In $(a, b)$ the cross-shore locations where $h(x)=2 d_{s}^{48 \mathrm{~h}}$ and $h(x)=\left(d_{s}^{48} \mathrm{~h}+\Delta d_{s}^{48 \mathrm{~h}} / 2\right)$ are indicated by black squares and circles, respectively, with a vertical dotted line in $(a)$. Also in $(b)$ is the far-field $E_{a}^{(z, f f)}$ scaling (6.4) in magenta for $h>3 d_{s}^{48 \mathrm{~h}}$ (triangle) and $\mathrm{d} T_{0} / \mathrm{d} z=0.25^{\circ} \mathrm{C} \mathrm{m}^{-1}$.

serves as an energy reservoir. Here, we evaluate whether the zero-crossing depth $d_{s}$ also characterizes $\mathcal{E}_{a}$, and thus the along-shore-averaged density $\bar{\rho}(x, z)$. First, the spatial structure of mean $\left\langle\mathcal{E}_{a}\right\rangle(x, z)(4.8)$ is evaluated for $\mathrm{d} T_{0} / \mathrm{d} z=0.25^{\circ} \mathrm{C} \mathrm{m}^{-1}$ (figure $10 a$ ). Consistent with its definition (4.8), elevated $\left\langle\mathcal{E}_{a}\right\rangle$ coincides with regions where $\langle\bar{T}\rangle$ isotherms are sloping (grey contours, figure $10 a)$ and is largest in the TRC region $(x>$ $\left.-4 L_{S Z}\right)$. A $\left\langle\mathcal{E}_{a}\right\rangle$ minimum at $z \approx-3.3 \mathrm{~m}$ coincides with the $t=48 \mathrm{~h}$ zero-crossing depth $d_{s}^{48 \mathrm{~h}}$ (diamond and dashed horizontal line, figure 10a). Above and below $z=-d_{s}^{48 \mathrm{~h}}$ are two $\left\langle\mathcal{E}_{a}\right\rangle$ reservoirs. The $z<-d_{s}^{48 \mathrm{~h}}$ reservoir occurs where $\langle\bar{T}\rangle>T_{s}$ and is concentrated where sloping isotherms intersect the bottom, near where $h=2 d_{s}^{48 \mathrm{~h}}$ (square and dotted vertical line, figure $10 a$ ). The $\left\langle\mathcal{E}_{a}\right\rangle$ is weak offshore of $x \approx-7 L_{S Z}$ and is roughly zero below $z=-3 d_{s}^{48 \mathrm{~h}}$. For $z>-d_{s}^{48 \mathrm{~h}}$, elevated $\left\langle\mathcal{E}_{a}\right\rangle$ is due to $\langle\bar{T}\rangle<T_{s}$. Onshore of where $h=2 d_{s}^{48 \mathrm{~h}}$ the temperature anomaly increases and $\left\langle\mathcal{E}_{a}\right\rangle \geq 1 \mathrm{~J} \mathrm{~m}^{-3}$ at the surface. Thus, $d_{s}^{48} \mathrm{~h}$ is the relevant vertical length scale describing the mean available potential energy response to TRCs.

The magnitude and cross-shore structure of $\left\langle\mathcal{E}_{a}\right\rangle$ reservoirs with varying $\mathrm{d} T_{0} / \mathrm{d} z$ are evaluated using depth-integrated available potential energy, defined as,

$$
E_{a}^{(z, t)}(x)=\int_{-h}^{\langle\bar{\eta}\rangle}\left\langle\mathcal{E}_{a}\right\rangle \mathrm{d} z .
$$


For all $\mathrm{d} T_{0} / \mathrm{d} z>0$, maximum $E_{a}^{(z, t)}$ occurs in the near-field region $\left(x \gtrsim-5 L_{S Z}\right)$ and increases from $0.5 \mathrm{~J} \mathrm{~m}^{-2}$ for the weakest $\mathrm{d} T_{0} / \mathrm{d} z$ to $3.25 \mathrm{~J} \mathrm{~m}^{-2}$ for the strongest stratification. The cross-shore location of $E_{a}^{(z, t)}$ moves onshore with increasing $\mathrm{d} T_{0} / \mathrm{d} z$, occurring roughly where $h(x)=2 d_{s}^{48} \mathrm{~h}$ (black squares, figure $10 b$ ), owing to contributions from both upper and lower $\left\langle\mathcal{E}_{a}\right\rangle$ reservoirs (black squares, figure $10 a$ ). There is an $E_{a}^{(z, t)}$ plateau onshore of $h \approx\left(d_{s}^{48 \mathrm{~h}}+\Delta d_{s}^{48 \mathrm{~h}} / 2\right)$ (black circles, figure 10a,b), because the upper- $\left\langle\mathcal{E}_{a}\right\rangle$ reservoir has a surface maximum and is weak below $z<-\left(d_{s}^{48 \mathrm{~h}}-\Delta d_{s}^{48 \mathrm{~h}} / 2\right)$ (upper dotted horizontal line, figure $10 a$ ). The far-field $E_{a}^{(z, t)}$ cross-shore decay length scale is similar to the far-field intrusion sloping isotherms (figure $3 d-f$ ), which increases with $\mathrm{d} T_{0} / \mathrm{d} z$. Thus, $d_{s}$ also describes the cross-shore distribution and magnitude of $E_{a}^{(z, t)}$, and will be used in subsequent sections to scale the inner-shelf mean stratification and circulation.

\section{Scaling the inner-shelf circulation and stratification response to TRC-induced mixing}

The sorted temperature anomaly analysis revealed the zero-crossing depth $d_{s}$ as the fundamental vertical length scale describing the inner-shelf mean specific and depth-integrated available potential energy $\left(\mathcal{E}_{a}(x, z)\right.$ and $\left.E_{a}^{(z, t)}(x)\right)$ response to TRC-induced mixing (figure 10). As available potential energy reservoirs are energy sources for inner-shelf mean circulation, cross-shore and along-shore velocity scalings are also anticipated to depend on $d_{s}$ as well as the initial stratification $\mathrm{d} T_{0} / \mathrm{d} z$ (or $N_{0}^{2}=$ $\left.g \alpha \mathrm{d} T_{0} / \mathrm{d} z\right)$. These are explored next.

\subsection{Self-similar near-field vertical stratification and cross-shore exchange flow}

The mean Lagrangian (Stokes + Eulerian) cross-shore velocity $\left\langle\bar{u}_{L}\right\rangle$ contributes to exchange between the near-field $(x \gtrsim-500 \mathrm{~m})$ and ambient inner-shelf farther offshore, or far-field $x \lesssim-500 \mathrm{~m}$. The near field is characterized by reduced vertical stratification, indicated by shoreward broadening isotherms (figure $3 d-f$ ), and an overturning streamfunction $\langle\bar{\Psi}\rangle$ with two counter-circulating cells (figure $3 g-i$ ). Over the 24-48 h averaging period, the near-field stratification and exchange flow are approximately constant. Here, we evaluate whether this dynamic equilibrium state is self-similar with respect to varying $\mathrm{d} T_{0} / \mathrm{d} z$. To account for variations in the cross-shore distribution of $\langle\bar{\Psi}\rangle$ and $\left\langle\bar{u}_{L}\right\rangle$ with $\mathrm{d} T_{0} / \mathrm{d} z$, the vertical length scale $d_{S}$ is used to re-define the boundary between near field and far field as $x=-L_{\text {mix }}$, where the depth is three times $d_{s}^{48 \mathrm{~h}}$,

$$
h\left(-L_{\text {mix }}\right)=3 d_{s}^{48 \mathrm{~h}},
$$

which is equivalent to $L_{\text {mix }}=3 d_{s}^{48 \mathrm{~h}} / \beta_{S Z}$ for planar beach slope $\beta_{S Z}$. The factor of 3 reflects that $d_{s}$ is the depth where the $x \geq-L_{m i x}$ area average of $T_{0}(z)$ is equivalent to $T_{0}\left(-d_{s}\right)$, see $(\mathrm{A} 1)$. The cross-shore location $x=-L_{m i x}$ roughly tracks the varying cross-shore location of inner-shelf $\langle\bar{\Psi}\rangle$ maxima for $\mathrm{d} T_{0} / \mathrm{d} z \geq 0.05^{\circ} \mathrm{C} \mathrm{m}^{-1}$ (triangle, figure $3 h, i)$. As the associated $d_{s}^{48 \mathrm{~h}}$ decreases from 4 to $2.8 \mathrm{~m}$ (figure $9 b$ ), the resulting maximum $h=12 \mathrm{~m}\left(L_{\text {mix }} \approx 6 L_{S Z}\right)$ for weakest $N_{0}$ decreases to $h=8 \mathrm{~m}\left(L_{\text {mix }} \approx 3 L_{S Z}\right)$ for highest $N_{0}$. Thus, the near-field $(x, z)$-area shrinks as stratification increases.

The vertical structures of $\langle\bar{N}\rangle$ and $\left\langle\bar{u}_{L}\right\rangle$ are scaled at $x=-L_{m i x}$. The mean stratification $\langle\bar{N}\rangle(z)$ is scaled by initial stratification $N_{0}$ and the non-dimensional depth $z / d_{s}^{48 \mathrm{~h}}$ is used 


\section{D.J. Grimes and F. Feddersen}

(a)

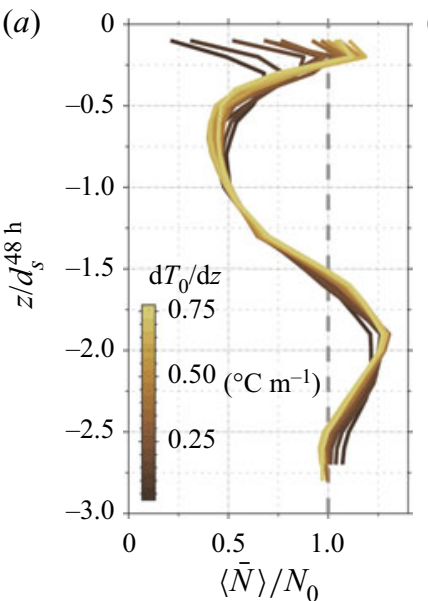

(b)

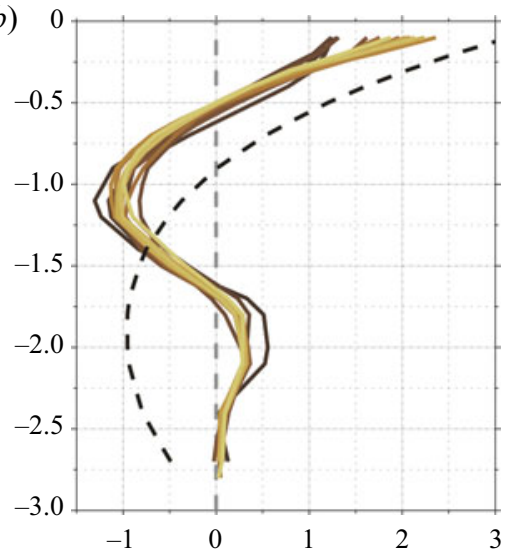

$\left\langle\bar{u}_{L}\right\rangle / \mathcal{U}$

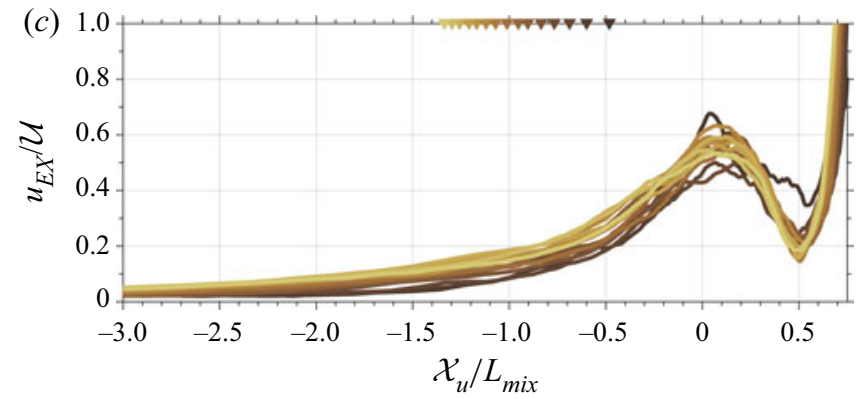

Figure 11. Non-dimensional mean (a) stratification $\langle\bar{N}\rangle / N_{0}$ and $(b)$ cross-shore Lagrangian velocity $\left\langle\bar{u}_{L}\right\rangle / \mathcal{U}$ vs non-dimensional depth $z / d_{s}^{48} \mathrm{~h}$ for $\mathrm{d} T_{0} / \mathrm{d} z \geq 0.05^{\circ} \mathrm{C} \mathrm{m}^{-1}$ at $x=-L_{m i x}$ (5.1), which varies from $h=12 \mathrm{~m}$ $\left(L_{m i x} \approx 6 L_{S Z}\right)$ to $h=8 \mathrm{~m}\left(L_{m i x} \approx 3 L_{S Z}\right)$ for the weakest to strongest $N_{0}$. In $(b)$ the unstratified $\mathrm{d} T_{0} / \mathrm{d} z=$ 0 simulation profile of $\left\langle u_{L}\right\rangle / U$ (dashed black line) is shown for $h\left(-6 L_{S Z}\right) \approx 12 \mathrm{~m}$ with $U=8 \times 10^{-4}$. (c) Non-dimensional inner-shelf cross-shore exchange velocity $\bar{u}_{E X} / \mathcal{U}$ (5.3) and (5.2) vs shifted non-dimensional cross-shore coordinate $\mathcal{X}_{u} / L_{\text {mix }}$ (5.4). In (c), the non-dimensional cross-shore locations of the far-field along-shore velocity maximum $x=-L_{\max }$ are shown on top as coloured down triangles.

to account for the shifting cross-shore location $L_{m i x}$ (figure 11a). The near-field $\left\langle\bar{u}_{L}\right\rangle(z)$ flow is related to $\mathrm{d} T_{0} / \mathrm{d} z$ using a baroclinic velocity scale (e.g. Manins 1976; Maxworthy \& Monismith 1988),

$$
\mathcal{U}=C_{0} N_{0} d_{s}
$$

with $C_{0}$ analogous to an intrusive gravity current (IGC) Froude number (e.g. Cheong, Kuenen \& Linden 2006; Bolster et al. 2008). Using $d_{s}^{48 \mathrm{~h}}$ in (5.2) with constant $C_{0}$ estimated by least-squares fit to the vertical minimum of $\left\langle\bar{u}_{L}\right\rangle(z)$ at $x=-L_{\text {mix }}$ (varying in the range $3-7 \mathrm{~mm} \mathrm{~s}^{-1}$ ) yields $C_{0} \approx 0.07$. The $x=-L_{\text {mix }}$ mean cross-shore velocity $\left\langle\bar{u}_{L}\right\rangle(z)$ is scaled by $\mathcal{U}$ and shown vs non-dimensional depth $z / d_{s}^{48 \mathrm{~h}}$ in figure $11(b)$.

Non-dimensional mean stratification profiles are self-similar below $z / d_{s}^{48} \mathrm{~h} \approx-0.35$, as the curves collapse (figure 11a). Sub-surface stratification is reduced for $-1<z / d_{s}^{48 \mathrm{~h}}<$ -0.5 , and slightly enhanced for $z / d_{s}^{48 \mathrm{~h}}<-1.5$. The minimum $\langle\bar{N}\rangle / N_{0} \approx 0.5$ occurs slightly above $z / d_{s}^{48 \mathrm{~h}}=-1$, consistent with the minimum $\partial_{z} \Delta T_{s}^{48 \mathrm{~h}}$ occurring shallower than $d_{s}^{48 \mathrm{~h}}$ (figure 9), potentially due to aliasing of $d_{s}$ time dependence. As $d_{s}$ growth is large until $t \approx 12 \mathrm{~h}$, fluid exported offshore earlier than $t=24 \mathrm{~h}$ would occupy a depth shallower than $z / d_{s}^{48 \mathrm{~h}}=-1$. Near-surface stratification is also reduced for small $\mathrm{d} T_{0} / \mathrm{d} z$, 
owing to the proximity to where the well-mixed fluid intersects the surface and due to larger near-surface $\left\langle\bar{K}_{V}\right\rangle$ for weak $N_{0}$ (e.g. figure $3 a$ ). Below $z / d_{s}^{48 \mathrm{~h}}=-1.5$, stratification is elevated, reaching $\langle\bar{N}\rangle / N_{0} \approx 1.25$ at $z / d_{s}^{48} \mathrm{~h} \approx-2$, consistent with the compressed $\langle\bar{T}\rangle$ isotherms and elevated r.m.s. $\{T\}$ at depth (figure $4 a$ ).

The non-dimensional cross-shore velocity $\left\langle\bar{u}_{L}\right\rangle / \mathcal{U}$ is also self-similar for $\mathrm{d} T_{0} / \mathrm{d} z \geq$ $0.05^{\circ} \mathrm{C} \mathrm{m}^{-1}$, and has a 3-layer exchange flow structure with near-surface and bottom onshore flow and offshore flow in between (figure $11 b)$. Near the surface $\left(z / d_{s}^{48 \mathrm{~h}}>-0.5\right.$ ), onshore flow is strong, with a maximum $\left\langle\bar{u}_{L}\right\rangle / \mathcal{U} \approx 2$. The maximum offshore flow occurs at $z / d_{s}^{48 \mathrm{~h}} \approx-1$, with $\left\langle\bar{u}_{L}\right\rangle / \mathcal{U} \approx-1$. Occurrence of the maximum offshore directed $\left\langle\bar{u}_{L}\right\rangle / \mathcal{U}$ flow where vertical stratification $\langle\bar{N}\rangle / N_{0}$ is minimum indicates sub-surface export of relatively low stratification (mixed) fluid, consistent with previous TRC exchange modelling (Kumar \& Feddersen 2017c; Grimes et al. 2020b) and differential mixing experiment and theory (e.g. Maxworthy \& Monismith 1988). Below $z / d_{s}^{48 \mathrm{~h}}=-1.5$, flow becomes onshore again with a weak maximum $\left\langle\bar{u}_{L}\right\rangle / \mathcal{U} \approx 0.25$ at $z / d_{s}^{48 \mathrm{~h}}=-2$. The relative strength of near-surface onshore transport $\left(z / d_{s}^{48 \mathrm{~h}}>-0.5\right)$ compared to sub-surface onshore transport $\left(z / d_{s}^{48} \mathrm{~h}<-1.5\right)$ implies stratified near-surface fluid is the dominant source to the TRC region. The 3-layer exchange flow for stratified cases is contrasted by the unstratified 2-layer $\left\langle\bar{u}_{L}\right\rangle / \mathcal{U}$ profile at $x=-6 L_{S Z}$ (black dashed, figure $11 b$ ), which is scaled by $\mathcal{U}=8 \times 10^{-4} \mathrm{~m} \mathrm{~s}^{-1}$ (roughly a factor of 3 smaller than weakest $N_{0}$ ) to give an offshore minimum $\left\langle\bar{u}_{L}\right\rangle / \mathcal{U} \approx-1$. The unstratified 2-layer structure is characteristic of Stokes drift and a quasi-uniform undertow velocity profile (e.g. Lentz et al. 2008; Hally-Rosendahl, Feddersen \& Guza 2014). The difference between 2-layer unstratified and 3-layer stratified $\left\langle\bar{u}_{L}\right\rangle$ demonstrates the different dynamics of the inner-shelf response.

The collapse of stratified equilibrium near-field $\langle\bar{N}\rangle / N_{0}$ and $\left\langle\bar{u}_{L}\right\rangle / \mathcal{U}$ profiles supports a dynamical similarity to IGC. Noting that the intrusion has $\langle\bar{N}\rangle / N_{0} \approx 1 / 2$ in (5.2), that is, $\mathcal{U}=C_{0} d_{s} N_{0} / 2$, increases the Froude number to $C_{0} \approx 0.13$ which is comparable to energy-conserving IGC from lock-release theory and experiments with $C_{0} \approx 0.25$. Based on dimensional analysis, the near-field velocity scaling $d_{S} N_{0}$ applies for both inertia and turbulent stress dominated exit flow, with the latter having $C_{0} \ll 1$, as observed in a laboratory experiment (Maxworthy \& Monismith 1988). The estimated advective speed $\mathcal{U} \approx 0.07\left(N_{0} d_{S}\right)$ is small relative to the background mode- 1 internal wave phase speed $\approx N_{0} d_{s}$ (e.g. White \& Helfrich 2008), suggesting radiated internal waves may contribute to the gravitational adjustment of the TRC-mixed fluid. Also, since the time averages span $t=24-48 \mathrm{~h}$, after an inertial period $\left(f^{-1}\right)$, the cross-shore speed may be reduced owing to the potential vorticity dynamics, as suggested by mean along-shore velocity $\langle\bar{v}\rangle$ (e.g. Thomas \& Linden 2007). The inner-shelf eddy activity in the TRC region $\left(x>-4 L_{S Z}\right)$ suggests viscous effects are non-negligible.

With the non-dimensional vertical structure at $x=-L_{m i x}$ well scaled, the cross-shore variation in baroclinic circulation $\langle\bar{\Psi}\rangle$ with initial stratification is evaluated using a cross-shore exchange velocity based on the mean cross-shore velocity $\left\langle\bar{u}_{L}\right\rangle$ (e.g. Grimes et al. 2020b),

$$
u_{E X}(x)=\frac{-2}{h+\langle\bar{\eta}\rangle} \int_{-h}^{\langle\bar{\eta}\rangle}\left\langle\bar{u}_{L}\right\rangle^{-}(x, z) \mathrm{d} z,
$$

where the vertical integral is restricted to offshore directed flow, indicated with superscript $\left({ }^{-}\right)$. For a bimodal vertical $\langle\bar{\Psi}\rangle(z)$, the offshore transport quantified with the vertical integral in (5.3) is equivalent to the difference between $\langle\bar{\Psi}\rangle$ vertical extrema, 


\section{D.J. Grimes and F. Feddersen}

$\max (\langle\bar{\Psi}\rangle)-\min (\langle\bar{\Psi}\rangle)$. Cross-shore exchange velocity $u_{E X}$ is a commonly used metric to evaluate cross-shore exchange potential (Suanda \& Feddersen 2015; Kumar \& Feddersen $2017 a, b)$. For $\mathrm{d} T_{0} / \mathrm{d} z \gtrsim 0.05^{\circ} \mathrm{C} \mathrm{m}{ }^{-1}, u_{E X}(x)$ has a local inner-shelf maximum near $x=-L_{m i x}$. A shifted non-dimensional near-field cross-shore coordinate is introduced,

$$
\mathcal{X}_{u} / L_{m i x}=\left(x+L_{m i x}\right) / L_{m i x},
$$

such that $\mathcal{X}_{u}>0$ is the near field and $\mathcal{X}_{u}<0$ is the far field (figure 11c).

The non-dimensional $u_{E X} / \mathcal{U}$ is self-similar with non-dimensional $\mathcal{X}_{u} / L_{\text {mix }}$ (figure $11 c$ ), indicating that $d_{s}$ is the fundamental vertical and horizontal (through $L_{m i x}$ ) length scale describing the near-field distribution of $\langle\bar{\Psi}\rangle$. Maxima of $u_{E X} / \mathcal{U}$ generally occur in the range $0<\mathcal{X}_{u} / L_{\text {mix }}<0.2$ with magnitude $\approx 1 / 2$. There is a local near-field exchange velocity minimum at $\mathcal{X}_{u} / L_{\text {mix }} \approx 0.5$, owing to closed inner-shelf and surf-zone $\langle\bar{\Psi}\rangle$ streamlines, partially due to the strong barotropic dominance of TRCs in this region (Kumar \& Feddersen 2017b). Offshore of the inner-shelf maximum, in the far-field $\left(\mathcal{X}_{u} / L_{m i x}<0\right)$, the horizontal decay length scale weakly increases relative to $L_{m i x}$ with increasing stratification, suggesting an additional far-field horizontal length scale that increases with $\mathrm{d} T_{0} / \mathrm{d} z$. Far-field circulation is evaluated in the next section.

Cross-shore exchange flows are common in many oceanographic settings, and either maintain or modify cross-shore tracer distributions (e.g. buoyancy, or pollutants). An alternative means of quantifying exchange flow strength is with a flushing time scale $\left(\mathcal{T}_{f}\right)$, a bulk measure of the transport time needed to fill a control volume (e.g. Lemagie \& Lerczak 2015). A near-field flushing time scale is estimated as the ratio of the cross-sectional near-field area, roughly $(9 / 2) d_{s}^{2} / \beta_{S Z}$ with bottom slope $\beta_{S Z}=0.025$, to the offshore directed transport, approximately $\left(d_{s} \mathcal{U}\right)$, giving,

$$
\mathcal{T}_{f} \sim \frac{9}{2} \frac{d_{s}^{2} / \beta_{S Z}}{d_{s}\left(C_{0} N_{0} d_{s}\right)}=\frac{9}{2}\left(\beta_{S Z} C_{0} N_{0}\right)^{-1} .
$$

Thus, increasing $\mathrm{d} T_{0} / \mathrm{d} z$ (larger $N_{0}$ ) decreases the flushing time $\mathcal{T}_{f}$ due to enhanced $\mathcal{U}$ and decreased near-field area (smaller $d_{s}$ ). Using modelled $d_{s}^{48} \mathrm{~h}, \mathcal{T}_{f}$ ranges from $5 \mathrm{~d}$ (smallest $N_{0}$ ) to $0.93 \mathrm{~d}$ (largest $N_{0}$ ). For reference, the unstratified 2-layer exchange flow requires $\approx 8 \mathrm{~d}$ to flush the near field.

\subsection{Self-similar far-field cross-shore stratification and along-shore thermal wind}

The strength of along-shore current $\langle\bar{v}\rangle$ increases with $\mathrm{d} T_{0} / \mathrm{d} z$ (figure $3 d-f$ ), forming a far-field $\left(x<-L_{m i x}\right)$ sub-surface positive along-shore current roughly centred between the broadening $\langle\bar{T}\rangle$ isotherms (figure $3 d-f$ ). Kumar \& Feddersen $(2017 b)$ found that, offshore of $\approx 4.5 L_{S Z}$, along-shore flow reached an approximate thermal wind balance,

$$
\frac{\partial\left\langle\bar{v}_{g}\right\rangle}{\partial z}=\frac{g \alpha}{f} \frac{\partial\langle\bar{T}\rangle}{\partial x} \equiv \frac{\left\langle\bar{M}^{2}\right\rangle}{f},
$$

where $\left\langle\bar{v}_{g}\right\rangle$ is the geostrophic component of along-shore velocity and $\left\langle\bar{M}^{2}\right\rangle$ the mean horizontal stratification. As the far-field intrusion has mean isotherms that slope upward (downward) above (below) $z=-d_{s}^{48 \mathrm{~h}}$ (e.g. figure 10a), thermal wind relation (5.6) also implies a sub-surface $\left\langle\bar{v}_{g}\right\rangle$ maximum, consistent with figure $3(j-l)$. A geostrophic horizontal length scale, based on the Rossby deformation radius $\left(L_{R}\right)$ and zero-crossing 
depth $\left(d_{s}\right)$,

$$
L_{R}=d_{s} N_{0} / f \text {, }
$$

is used to scale the far-field response. In contrast to $L_{\text {mix }}$, which decreases weakly with increasing $\mathrm{d} T_{0} / \mathrm{d} z$, the magnitude of $L_{R}(5.7)$ using $d_{s}^{48} \mathrm{~h}$ increases with $\mathrm{d} T_{0} / \mathrm{d} z$, ranging from $\approx 260 \mathrm{~m}$ to $\approx 1300 \mathrm{~m}$. The far-field location of maximum mean along-shore velocity, denoted $x=-L_{\max }$, is well represented by,

$$
L_{\text {max }}=C_{\text {mix }} L_{\text {mix }}+C_{R} L_{R},
$$

with constant $C_{\text {mix }}=1.23$ and $C_{R}=0.3$, and varies between $L_{\max }=800$ and $900 \mathrm{~m}$ (not shown). Vertical profiles of $\left\langle\bar{M}^{2}\right\rangle(z)$ and $\langle\bar{v}\rangle(z)$ at $x=-L_{\max }$ are used to evaluate the structure and scale of the far-field response.

An along-shore velocity $\langle\bar{v}\rangle$ scaling based on the thermal wind relation (5.6) is formed by relating the far-field horizontal temperature gradient to the initial stratification using the geostrophically balanced isotherm slope scaling (Lentz \& Chapman 2004),

$$
\mathrm{d} z / \mathrm{d} x=\left\langle\bar{M}^{2}\right\rangle /\left\langle\bar{N}^{2}\right\rangle \sim f / N_{0},
$$

and assuming self-similar far-field vertical stratification $\langle\bar{N}\rangle \propto N_{0}$, such that,

$$
\left\langle\bar{M}^{2}\right\rangle=C_{1} f N_{0}
$$

The isotherm slope scaling (5.9) implies a local far-field intrusion aspect ratio proportional to $d_{S} / L_{R}$. Thus, $L_{R}$ scales the horizontal distance over which far-field isotherms broaden. A geostrophic velocity scale $\mathcal{V}$ is formed by substituting (5.10) in (5.6) and assuming $d_{S}$ parameterizes the vertical scale over which $\langle\bar{v}\rangle$ increases to $\mathcal{V}$ giving,

$$
\mathcal{V} \approx C_{1} d_{s} N_{0},
$$

with constant $C_{1}=0.26$ determined using $d_{s}^{48 \mathrm{~h}}$ in (5.11) through least-squares fit to the $z$-maximum $\langle\bar{v}\rangle$ at $x=-L_{\max }$.

Non-dimensional mean horizontal stratifications $\left\langle\bar{M}^{2}\right\rangle /\left(C_{1} f N_{0}\right)$ at $x=-L_{\max }$ generally collapse vs non-dimensional depth $z / d_{s}^{48} \mathrm{~h}$ (figure $12 a$ ). Near the surface, $\left\langle\bar{M}^{2}\right\rangle /\left(C_{1} f N_{0}\right)$ is negative and has an $O(1)$ local minimum at $z / d_{s}^{48 \mathrm{~h}} \approx-0.3$. Over $-2<z / d_{s}^{48 \mathrm{~h}}<$ $-0.75,\left\langle\bar{M}^{2}\right\rangle /\left(C_{1} f N_{0}\right)$ is positive, with an $O(1)$ local maximum at $z / d_{s}^{48} \mathrm{~h} \approx-0.8$. Below $z / d_{s}^{48 \mathrm{~h}}=-2,\left\langle\bar{M}^{2}\right\rangle /\left(C_{1} f N_{0}\right)$ becomes weakly negative again. Using the near-field minimum stratification $\langle\bar{N}\rangle / N_{0} \approx 1 / 2$ in (5.9) and (5.10) gives a theoretical estimate for $C_{1}=1 / 4$ with extrema of $\left\langle\bar{M}^{2}\right\rangle /\left(C_{1} f N_{0}\right) \approx \pm 1$, consistent with the fit $C_{1}=0.26$ and the magnitude of non-dimensional extrema (figure 12a). The collapse is poorer for the weakest stratification runs with $\mathrm{d} T_{0} / \mathrm{d} z \leq 0.1^{\circ} \mathrm{C} \mathrm{m}^{-1}$. Non-dimensional mean along-shore velocity $\langle\bar{v}\rangle / \mathcal{V}$ at $x=-L_{\max }$ also collapses (figure $12 b$ ), with vertical slope generally of the same sign as $\left\langle\bar{M}^{2}\right\rangle$, consistent with the thermal wind relation (5.6). There is a weak near-surface $\langle\bar{v}\rangle / \mathcal{V}$ minimum at $z / d_{s}^{48 \mathrm{~h}} \approx-0.25$. Below $z / d_{s}^{48 \mathrm{~h}} \approx-0.75,\langle\bar{v}\rangle / \mathcal{V}$ becomes positive and has a sub-surface $O(1)$ maximum at roughly $z / d_{s}^{48 \mathrm{~h}}=-1$ and then decreases again as $\left\langle\bar{M}^{2}\right\rangle$ becomes positive. For $z / d_{s}^{48 \mathrm{~h}}<-1.75,\langle\bar{v}\rangle / \mathcal{V}$ is again weakly negative and tends toward zero as $z / d_{s}^{48} \mathrm{~h}$ decreases below -3 . Consistency of $\left\langle\bar{M}^{2}\right\rangle /\left(C_{1} f N_{0}\right)$ and $\langle\bar{v}\rangle / \mathcal{V}$ indicates the far-field mean along-shore velocity has reached an approximate geostrophic balance with the mean cross-shore horizontal stratification, with $x=-L_{\max }$ isotherm slopes well parameterized by $\mathrm{d} z / \mathrm{d} x \sim f / N_{0}$ (5.9). 


\section{D.J. Grimes and F. Feddersen}
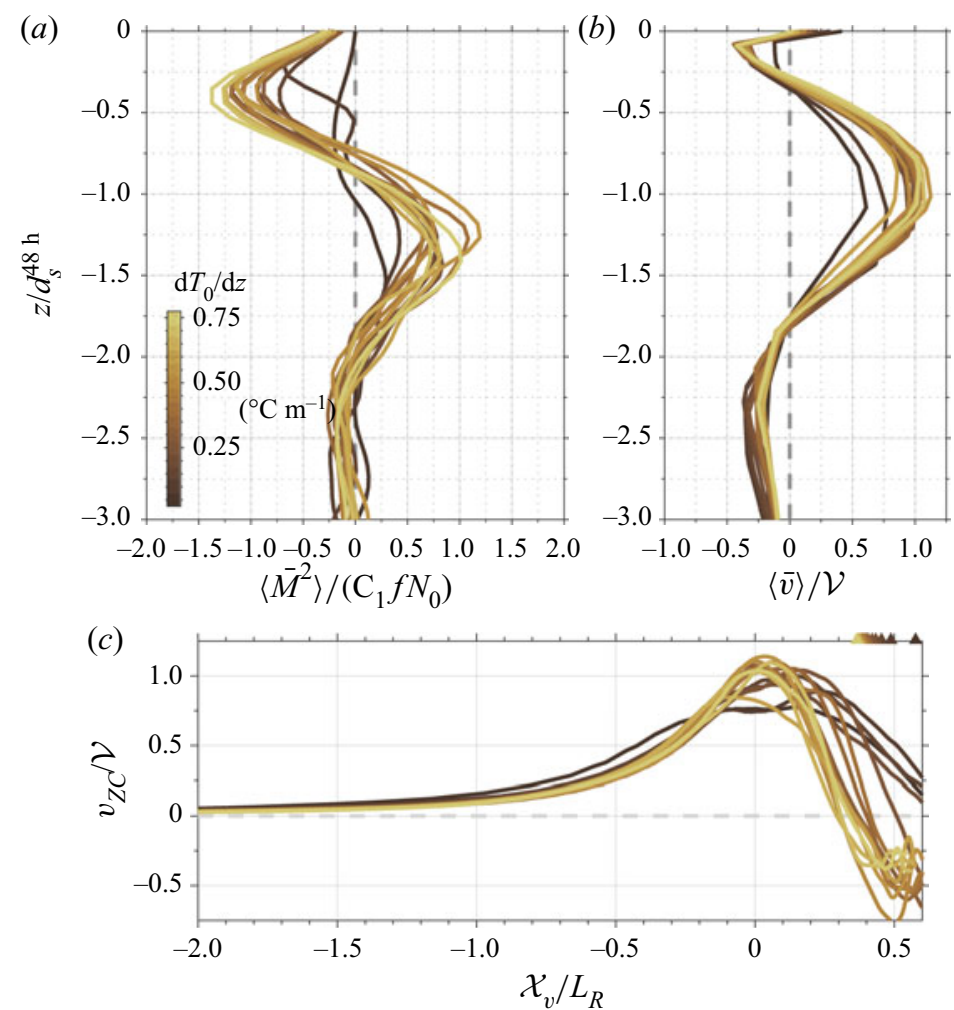

Figure 12. (a) Non-dimensional mean horizontal stratification $\left\langle\bar{M}^{2}\right\rangle /\left(C_{1} f N_{0}\right)(5.10)$ and $(b)$ non-dimensional mean along-shore velocity $\langle\bar{v}\rangle / \mathcal{V}(5.11)$ vs non-dimensional depth $z / d_{s}^{48 \mathrm{~h}}$ for $\mathrm{d} T_{0} / \mathrm{d} z \geq 0.05^{\circ} \mathrm{C} \mathrm{m}^{-1}$ at $x=$ $-L_{\max }$ (5.8) which varies from $x=-800$ to $-900 \mathrm{~m}$. (b) Non-dimensional inner-shelf zero-crossing depth mean along-shore velocity $v_{Z C} / \mathcal{V}(5.12)$ and (5.11) vs shifted non-dimensional cross-shore coordinate $\mathcal{X}_{v} / L_{R}$ (5.13). In (c), the non-dimensional cross-shore location of the near-field/far-field division $x=-L_{m i x}$ is shown on top as coloured up-triangles.

The far-field cross-shore structure of $\langle\bar{v}\rangle$ is evaluated using a cross-shore transect at the zero-crossing depth, denoted,

$$
v_{Z C}(x)=\langle\bar{v}\rangle\left(x,-d_{s}^{48 \mathrm{~h}}\right) .
$$

Based on the isotherm slope scaling (5.9) at $x=-L_{\max }$, a far-field shifted non-dimensional cross-shore coordinate is introduced,

$$
\mathcal{X}_{v} / L_{R}=\left(x+L_{\max }\right) / L_{R},
$$

where $\mathcal{X}_{v}=0$ is the maximum along-shore current location. Non-dimensional $v_{Z C} / \mathcal{V}$ generally collapses using $\mathcal{X}_{v} / L_{R}$ (figure $12 c$ ), particularly for $\mathcal{X}_{v} / L_{R}<0$. The non-dimensional zero-crossing depth velocity $v_{Z C} / \mathcal{V}$ decays to near zero by $\mathcal{X}_{v} / L_{R} \approx$ -2 , indicating $L_{R}$ accurately scales the offshore decay of the geostrophic current. Moving onshore from $\mathcal{X}_{v} / L_{R}=0$, toward the near field at $\mathcal{X}_{u}=0$ (up-triangles, top of figure $12 c$ ), the curves separate with lower stratification having larger shoreward decay scale, consistent with transition to a near-field horizontal scale characterized by $L_{m i x}$. Thus, inner-shelf response to TRC mixing is determined by two length scales, $L_{\text {mix }} \sim d_{S} / \beta_{S Z}$ in the near field, and Rossby deformation radius $L_{R} \sim d_{S} N_{0} / f$ in the far field, both based on the fundamental vertical scale $d_{s}$. The $L_{R}$ dependence suggests that the offshore extent 
of the far-field intrusion is larger at lower latitudes (smaller $f$ ). As the intrusion growth is constrained by the near-field exchange flow (e.g. Maxworthy \& Monismith 1988), the time scale for the far-field intrusion to reach length $L_{R}$ roughly scales as $L_{R} / \mathcal{U} \sim f^{-1}$, indicating that, at lower latitudes, geostrophic balance may not occur over the $24-48 \mathrm{~h}$ time scale considered here.

\subsection{Scaling the domain-integrated potential energy growth rate}

As the surf-zone to inner-shelf kinetic energy flux $F_{k^{\prime}}^{(s z)}$ is independent of $N_{0}$, the near-field mean exchange flow and stratification scalings are used to relate the continuous TRC-region mixing to the domain-integrated potential energy growth rate $\left\langle\partial_{t} E_{p}^{(x, z)}\right\rangle$, or equivalently $\gamma_{E}$ (4.11). The scaling analysis assumes that the near-field exchange flow $\mathcal{U}$, zero-crossing depth $d_{s}$ and stratification $N$ are constant, implying that $\left\langle\partial_{t} E_{p}^{(x, z)}\right\rangle$ is representative of the increase in far-field $\left(\mathcal{X}_{u}<0\right)$-integrated $E_{p}^{(x, z)}$. With this assumption, and noting that far-field vertical mixing is relatively weak (figure $3 a-c),\left\langle\partial_{t} E_{p}^{(x, z)}\right\rangle$ can be approximated with a cross-shore advective buoyancy flux between the near-field and far-field regions, or,

$$
\left\langle\partial_{t} E_{p}^{(x, z)}\right\rangle \sim \varrho \int_{-3 d_{s}}^{0} z^{\prime} \mathcal{B}\left(z^{\prime}\right) \mathrm{d} z^{\prime},
$$

where $\mathcal{B}(z)=-g \varrho^{-1}\langle\overline{u \rho}\rangle$ is the vertically varying mean cross-shore buoyancy flux at $\mathcal{X}_{u}=0$. Leveraging the self-similar vertical stratification and cross-shore exchange flow, the buoyancy flux $(\mathcal{B})$ is approximated as constant,

$$
\mathcal{B} \sim \mathcal{U} d_{s} N_{0}^{2}
$$

where $\left(d_{S} N_{0}^{2}\right)$ parameterizes the buoyancy anomaly between onshore and offshore directed transports. Lastly, scaling the vertical integral of $\left(z^{\prime} \mathrm{d} z^{\prime}\right)$ in (5.14) with $d_{s}^{2}$ gives,

$$
\begin{aligned}
\left\langle\partial_{t} E_{p}^{(x, z)}\right\rangle & \sim \varrho \mathcal{U} d_{s}^{3} N_{0}^{2}, \\
& \propto \varrho d_{s}^{4} N_{0}^{3} .
\end{aligned}
$$

Using modelled $d_{S}^{48 \mathrm{~h}}$ and dimensionless proportionality constant 0.05 in (5.16), scaling estimates of $\gamma_{E}$ generally reproduce the modelled $\mathrm{d} T_{0} / \mathrm{d} z$ dependence (crosses, figure 8), particularly for $\mathrm{d} T_{0} / \mathrm{d} z \geq 0.1^{\circ} \mathrm{C} \mathrm{m}^{-1}$. The consistency of scaling (5.16) supports the assumption of steady near-field $\mathcal{U}$ and $d_{s}$, and dynamic equilibrium between the cross-shore buoyancy flux at $x=-L_{\text {mix }}$ (5.15) and the near-field mixing. Conceptually, the exchange flow, scaled by $\mathcal{U}$, continuously supplies the TRC region with stratified fluid from offshore, such that, when mixed, the cross-shore pressure gradient and near-field available potential energy (figure 10) are maintained. Thus, the surf-zone and TRC mixing-driven circulation is largely self-sustaining, provided sufficient supply of stratified fluid to maintain it. Consequently, the approximately geostrophic far-field intrusion slowly grows throughout the averaging period. At very long time scales, it is not clear how the far-field dynamics impacts the near-field exchange flow. 


\section{D.J. Grimes and F. Feddersen}

\section{Discussion}

\subsection{Relating zero-crossing depth $d_{s}$ to initial stratification}

The specific background potential energy distribution, quantified using the sorted temperature anomaly $\Delta T_{s}$, was characterized by the zero-crossing depth $d_{s}$ (figure 9). The zero-crossing depth $d_{s}$ is also the fundamental length scale describing the inner-shelf available potential energy $\left(\left\langle\mathcal{E}_{a}(x, z)\right\rangle\right.$ and $E_{a}^{(z, t)}$, figure 10), the self-similar near-field vertical stratification and cross-shore velocity $\left(\langle\bar{N}\rangle / N_{0}\right.$ and $\left\langle\bar{u}_{L}\right\rangle / \mathcal{U}$, figure 11) and far-field horizontal stratification and along-shore velocity $\left(\left\langle\bar{M}^{2}\right\rangle /\left(C_{1} f N_{0}\right)\right.$ and $\langle\bar{v}\rangle / \mathcal{V}$, figure 12), as well as the domain-averaged conversion efficiency ( $\gamma_{E}$, figure 8$)$. As $d_{S}$ was estimated from the simulations, it is not clear how $d_{S}$ depends on the initial stratification and the mixing associated with wave and TRC forcing. Here, relatively simple energetics arguments are used to derive a scaling for $d_{s}$.

To derive analytical expressions for the sorted temperature anomaly $\Delta T_{S}$ and zero-crossing depth $d_{s}$, a simplified domain geometry is used with constant bathymetric slope $h(x)=-\beta x$ that extends offshore to $x=-L_{x}$ with maximum depth $h_{\max }=\beta L_{x}$ (figure 13a). Surf-zone and TRC-induced mixing is also idealized by homogenizing the constant stratification initial temperature $T_{0}(z)(2.1)$ within the wedge formed by a vertical offshore front at $x=-L_{m i x}$ (figure 13a). Derivations are given in Appendix A. The idealized well-mixed wedge can be thought of as the short time scale effect of the TRC-region mixing (e.g. $t<3 \mathrm{~h}$ figure $7 a, b$ ), before the development of inner-shelf $\bar{\Psi}(x, z, t)$ and $\bar{v}(x, z, t)$. The well-mixed fluid has uniform temperature $T_{m i x}=T_{0}\left(-d_{s}\right)$, where $d_{s}=\beta L_{\text {mix }} / 3$ (A1) is the $\Delta T_{s}$ zero-crossing depth for the idealized well-mixed wedge (dashed, figure $13 a$ ). With these assumptions, analytical expressions for the sorted temperature profile $T_{s}(z)$ (A13), and $\Delta T_{s}(z)$ (right of figure 13a), vary depending on $d_{s}$ and with the parameter $\epsilon_{z}=d_{s} / h_{\max }$, quantifying the ratio of the zero-crossing depth to the maximum offshore domain depth. The idealized $\Delta T_{s}$ profile is qualitatively consistent with the modelled $\Delta T_{S}$ curves in many respects, with $\Delta T_{S}$ extrema above and below $d_{s}$ and $\Delta T_{s}=0$ below $z=-3 d_{s}=h_{\text {mix }}$ (right of figure 13a; and figure 9). For the idealized $T_{s}$ profile, the region $\left(-d_{s}-\Delta d_{s} / 2\right)<z<\left(-d_{s}+\Delta d_{s} / 2\right)$ has minimum possible $\partial T_{s} / \partial z=0$ and $\partial_{z} \Delta T_{s}=-\mathrm{d} T_{0} / \mathrm{d} z$ owing to the idealized wedge being perfectly mixed, in contrast to the weaker $t=48 \mathrm{~h}$ modelled slope $\partial_{z} \Delta T_{s}^{48} \mathrm{~h} \approx-0.5 \mathrm{~d} T_{0} / \mathrm{d} z$ (figure $9 a$ ).

Here, the potential energy change $\left(E_{p}^{\text {mix }}\right)$ associated with homogenizing $x>-L_{\text {mix }}$ is used to relate the modelled zero-crossing depth $d_{s}$ to the initial stratification $\mathrm{d} T_{0} / \mathrm{d} z$. The change is assumed to depend on the eddy kinetic energy flux from the surf zone to inner shelf, i.e.

$$
E_{p}^{m i x} \propto \tau_{m i x} F_{k^{\prime}}^{(s z)}
$$

where $\tau_{m i x}$ is the idealized wedge mixing time scale and $F_{k^{\prime}}^{(s z)} \equiv F_{k^{\prime}}\left(x=-L_{S Z}\right)$. The time scale $\tau_{\text {mix }}$ is assumed to depend on TRC characteristics (independent of $\mathrm{d} T_{0} / \mathrm{d} z$ ) and $O(1 \mathrm{~h}$ ) based on the $E_{k^{\prime}}^{(x, z)}$ spin-up (figure $\left.6 b\right)$. Thus, $\tau_{\text {mix }}$ is short relative to the $\mathcal{T}_{f} \sim O(1 \mathrm{~d}$ ) (5.5) flushing time scale that would modify the distribution of idealized well-mixed fluid. The modelled conversion efficiency $\gamma_{E}$ was well represented by cross-shore buoyancy flux scaling (5.16) assuming constant in time near-field $E_{p}^{(x, z)}$. As $F_{k^{\prime}}$ is constant across simulations (figure $6 c$ ), (6.1) implies $E_{p}^{m i x}$ is constant in time, consistent with the $\gamma_{E}$ scaling. The potential energy change in forming the idealized well-mixed wedge with 

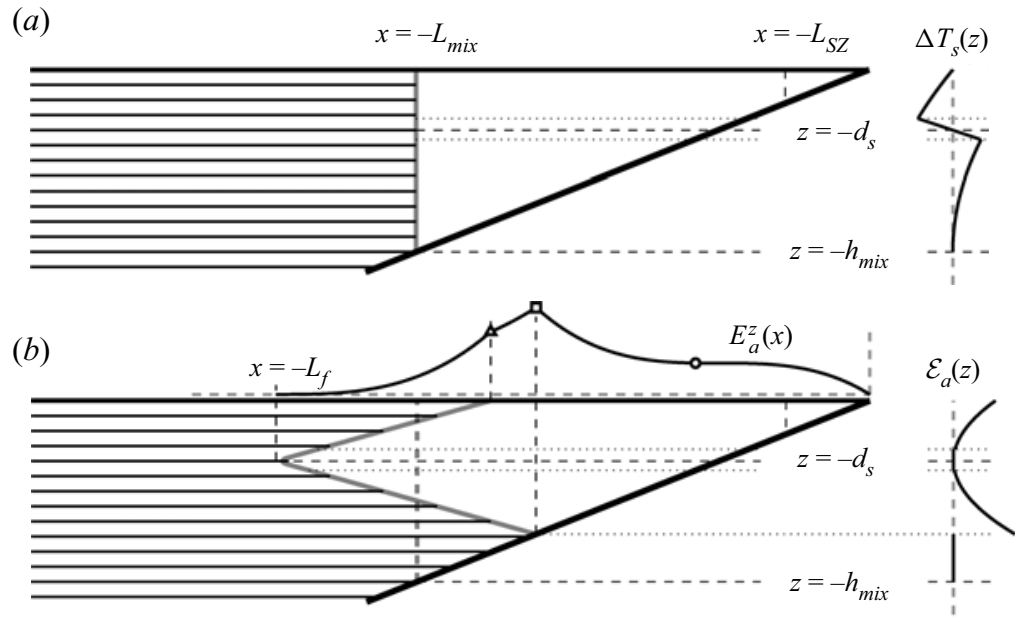

Figure 13. (a) Idealized well-mixed wedge driven by TRC-induced mixing of temperature onshore of $x=$ $-L_{\text {mix }}$ with the associated sorted temperature anomaly $\Delta T_{s}$ (right). (b) Idealized offshore intrusion of well-mixed fluid, with the vertical structure of $\mathcal{E}_{a}(z)$ for the well-mixed wedge (right) and the cross-shore $E_{a}^{z}(x)$ structure (above). In $(a, b)$, the zero-crossing depth $d_{s}$ and mixed region depth $h_{m i x}=3 d_{s}$ are indicated with horizontal dashed black line, and the location of the upper and lower bounds $\left(d_{s} \pm \Delta d_{s} / 2\right)$ (denoted $d_{s}^{+/-}$in Appendices A-C) of the unstratified $T_{s}(z)$ region are indicated with horizontal dotted lines. In $(b)$, the cross-shore location of the surface front (triangle), bottom front (square) and plateau (circle) are shown, roughly corresponding to the markers in figure $10(b)$. The offshore apex at $x=-L_{f}$ is also indicated.

$T_{\text {mix }}=T_{0}\left(-d_{s}\right)$ and linear temperature-dependent equation of state (2.2),

$$
\begin{aligned}
E_{p}^{m i x} & =\int_{-L_{\text {mix }}}^{0} \int_{-h(x)}^{0} g z\left[\rho_{0}\left(-d_{s}\right)-\rho_{0}(z)\right] \mathrm{d} z \mathrm{~d} x, \\
& =\frac{1}{36} \varrho \beta^{3} N_{0}^{2} L_{m i x}^{4} \equiv \frac{9}{4} \varrho \beta^{-1} N_{0}^{2} d_{s}^{4} .
\end{aligned}
$$

As the idealized offshore region $x<-L_{\text {mix }}$ is unchanged, $E_{p}^{\text {mix }}$ is both a measure of the potential energy change of the idealized wedge and the entire domain. Combining (6.2) with (6.1) gives,

$$
\left\{d_{s}, L_{m i x}\right\} \propto\left(\tau_{m i x} F_{k^{\prime}}^{(s z)}\right)^{1 / 4}\left(\varrho N_{0}^{2}\right)^{-1 / 4},
$$

consistent with the equilibrium mixed layer depth scaling $d \sim N_{0}^{-1 / 2}$ of Maxworthy \& Monismith (1988). As such, $d_{s}$ is expected to decrease as stratification increases, and increase with increasing $F_{k^{\prime}}^{(s z)}$. The $\mathrm{d} T_{0} / \mathrm{d} z \geq 0.05^{\circ} \mathrm{C} \mathrm{m}^{-1}$ model $d_{s}^{48 \mathrm{~h}}$ (figure $9 b$ ) is consistent with modified $(6.3),\left(d_{s}^{48 \mathrm{~h}}-d_{0}\right) \propto N_{0}^{-1 / 2}$, with $d_{0}=1.75 \mathrm{~m}$ and proportionality constant $0.22 \mathrm{~m} \mathrm{~s}^{-1 / 2}$, giving a skill of 0.98 (not shown). The additional length scale $d_{0}$ for large stratification may depend on the surf-zone depth $h_{S z}=\beta_{S Z} L_{S Z}$, such that, if inner-shelf mixing is suppressed to the point of $d_{s} \approx h_{s z}$, then the only source of mixing is the turbulent surf zone. The decrease in the $(x, z)$-extent of elevated $\left\langle\bar{K}_{V}\right\rangle$ with increasing $\mathrm{d} T_{0} / \mathrm{d} z$ (figure $3 a-c$ ) is consistent with a decrease in both $\left\{d_{s}, L_{\text {mix }}\right\}$ with $\mathrm{d} T_{0} / \mathrm{d} z$, as are the onshore movement of $u_{E X}$ and $E_{a}^{(z, t)}$ maxima. Thus, the vertical length scale $d_{s}$ delimiting the near field, $h<3 d_{s}$, and determining the vertical structure $z / d_{s}$ of the inner-shelf response is related to the TRC eddy kinetic energy flux from the surf zone 


\section{D.J. Grimes and F. Feddersen}

to the inner shelf $F_{k^{\prime}}^{(s z)}$ and initial stratification $N_{0}$ through the work done in mixing an idealized well-mixed wedge. However, the idealized well-mixed wedge does not account for several dynamical aspects of the mean inner-shelf response to TRC-region mixing, e.g. the far-field intrusion, which are addressed next.

\subsection{Idealized cross-shore $E_{a}^{(z, t)}(x)$ structure}

The idealized well-mixed wedge $x=-L_{\text {mix }}$ vertical front (figure $13 a$ ) is gravitationally unstable and adjusts to form the far-field intrusion characterized by mean $\langle\bar{T}\rangle$ isotherms that broaden toward shore (figure $3 d-f$ ). The far-field isotherm slope scaling $\mathrm{d} z / \mathrm{d} x=f / N_{0}$ (5.10, figure 12a) suggests that, in contrast to $d_{s}$ which decreases with increasing $\mathrm{d} T_{0} / \mathrm{d} z$, the offshore extent of TRC-mixed water mass increases with $\mathrm{d} T_{0} / \mathrm{d} z$, owing to larger $L_{R}$. Here, the vertical $x=-L_{\text {mix }}$ offshore front is transformed to an idealized far-field intrusion sloping symmetrically about $z=-d_{s}$ (figure 13b) to illustrate how salient features of the cross-shore distribution of $E_{a}^{(z, t)}(x)$ (and thus the mean density $\langle\bar{\rho}\rangle(x, z)$ ) relate to $d_{s}$ and $L_{R}$. Holding $A_{\text {mix }}=(9 / 2) d_{S} / \beta$ and thus $d_{s}$ constant for simplicity, the apex of the idealized intrusion at $x=-L_{f}$ moves offshore as the intrusion slope $\mathrm{d} z / \mathrm{d} x=$ $f / N_{0}$ decreases (B7)-(B11). For simplicity, isotherm adjustment outside of the idealized intrusion is neglected, meaning the adiabatic transformation from idealized well-mixed wedge to idealized intrusion decreases the domain-integrated available potential energy, qualitatively consistent with conversion to inner-shelf mean circulation.

Here, $\mathcal{E}_{a}$ and $E_{a}^{z}$ scalings for the idealized intrusion (figure 13b) are summarized, and detailed derivations are found in Appendix B. To leading order in $\epsilon_{z}=d_{s} / h_{\max }$, the specific available potential energy within the idealized well-mixed wedge and intrusion scales as $\mathcal{E}_{a}(z) \propto \varrho N_{0}^{2}\left(z+d_{s}\right)^{2}(\mathrm{~B} 1)$, and is concentrated in two reservoirs above and below $d_{s}$ (right, figure $13 b$ ), roughly consistent with the modelled $\left\langle\mathcal{E}_{a}\right\rangle$ (figure $10 a$ ). Vertically integrating $\mathcal{E}_{a}(z)$ over the well-mixed wedge and intrusion gives $E_{a}^{z}(x)$, which at leading order is proportional to $\varrho N_{0}^{2} d_{s}^{3}$ with cubic dependence on sloping boundaries, e.g. $h(x)$ in (B4) and (B12). Similar to the modelled $E_{a}^{(z, t)}(x)$ (figure 10b), moving away from shore $E_{a}^{z}(x)$ plateaus where $\left(d_{s}-\Delta d_{s} / 2\right)<h(x)<\left(d_{s}+\Delta d_{s} / 2\right)$ (dotted lines horizontal lines, figure $13 b)$ then increases again for $h(x)>\left(d_{s}+\Delta d_{s} / 2\right)$ (circle, figure 13b). For the idealized well-mixed intrusion, maximum $E_{a}^{z}(x) \propto \varrho N_{0}^{2} d_{s}^{3}$ occurs at $h<3 d_{s}$ where the vertical thickness of the well-mixed fluid is largest (square, figure 13b). The maximum vertical thickness depends on where the idealized well-mixed intrusion intersects the surface and bottom (triangle and square, figure $13 b$ ) and varies with $f / N_{0}$ (B12). For large $N_{0}$, the front locations approach the TRC region and surf zone, where strong mixing would limit further onshore progression, requiring additional constraints not considered here. The modelled $E_{a}^{(z, t)}$ maxima occur near $h(x)=2 d_{s}^{48 \mathrm{~h}}$ (squares, figure $10 b$ ), and are well represented by the leading-order idealized intrusion dependence $\max \left\{E_{a}^{z}(x)\right\} \propto \varrho N_{0}^{2} d_{s}^{3}$, with best fit proportionality constant 0.12 for all $\mathrm{d} T_{0} / \mathrm{d} z>0$, giving $r^{2}=0.99$ and skill score 0.97. Note, this leading-order dependence is consistent with the cross-shore advective buoyancy flux scaling (5.16), which can be written as $\left\langle\partial_{t} E_{p}^{(x, z)}\right\rangle \propto \mathcal{U} \max \left\{E_{a}^{z}(x)\right\}$.

Restricting attention to the far-field region, defined here as where the idealized intrusion is symmetric about $z=-d_{s}$ with vertical thickness $H(x)=2 f / N_{0}\left(x+L_{f}\right)$ (B6) (offshore of $\triangle$, figure $13 b$ ), the idealized intrusion $E_{a}^{z}$ varies as (B13),

$$
E_{a}^{(z, f f)} \propto \varrho N_{0}^{2} d_{s}^{3}\left[1+\left(x-x_{0}\right) / L_{R}\right]^{3},
$$


with $x_{0}$ corresponding to the triangle in figure 13(b) and using the Rossby deformation radius $L_{R}=d_{S} N_{0} / f$. The functional dependence (6.4) is shown in figure $10(b)$ (dashed magenta) for $\mathrm{d} T_{0} / \mathrm{d} z=0.25^{\circ} \mathrm{C} \mathrm{m}^{-1}$, with $x_{0}=-L_{\text {mix }}$ (5.1) and $L_{R}=d_{s}^{48} \mathrm{~h}_{0} / f(5.7)$, and largely scales the offshore decay in the modelled $E_{a}^{(z, t)}$. However, the parameters $x_{0}$ and proportionality constant $(0.1)$ vary with $\mathrm{d} T_{0} / \mathrm{d} z$ due to additional near-field dynamics. The $L_{R}$ far-field decay length scale is also consistent with the distribution of $v_{Z C} / \mathcal{V}$ (figure $12 c$ ). Thus, the idealized well-mixed intrusion is qualitatively consistent with the modelled isotherms broadening with aspect ratio $d_{s} / L_{r}$ and varying with initial stratification $N_{0}$, and zero-crossing depth $d_{s}$ (a function of both $\mathrm{d} T_{0} / \mathrm{d} z$ and $F_{k^{\prime}}^{(s z)}$ ).

\subsection{Scaling incident wave effects on stratified inner-shelf response}

Using a simplified representation of TRC mixing of inner-shelf temperature, the length scale $d_{s}$ characterizing the cross-shore and vertical structure of inner-shelf response, was related to the work done in mixing through the cross-shore kinetic energy flux $F_{k^{\prime}}$ over a short time scale $\tau_{\text {mix }}(\S 6.1)$. The incident wave forcing was kept constant for all simulations and $F_{k^{\prime}}$ was essentially unaffected by the large range of $\mathrm{d} T_{0} / \mathrm{d} z(\$ 4.1)$. Thus, the derived near-field exchange velocity scaling $\mathcal{U}(5.2)$ and far-field along-shore thermal wind scaling $\mathcal{V}(5.11)$ do not explicitly relate the inner-shelf circulation $\left(\left\langle\bar{u}_{L}\right\rangle\right.$ and $\left.\langle\bar{v}\rangle\right)$ and stratification $\left(\langle\bar{N}\rangle\right.$ and $\left.\left\langle\bar{M}^{2}\right\rangle\right)$ response to the incident waves forcing TRCs. However, these scalings implicitly depend on the magnitude of wave and TRC forcing, through the surf-zone to inner-shelf eddy kinetic energy flux $F_{k^{\prime}}^{(s z)}$ and zero-crossing depth $d_{s}$. Based on energy arguments, the zero-crossing depth roughly scales as $d_{s} \propto\left(F_{k^{\prime}}^{(s z)}\right)^{1 / 4}$ (6.3). Here, we explore how variations in incident wave conditions, e.g. wave height $H_{S z}$, deep-water wavelength $L_{\infty}=g T_{p}{ }^{2} /(2 \pi)$ or peak period $T_{p}$ and directional spread $\sigma_{\theta}$, affect $F_{k^{\prime}}$ and in turn the zero-crossing depth $d_{s}$. For simplicity, the surf-zone depth $h_{s z}=\left(\beta_{S Z} L_{S Z}\right)$ is determined from wave height using constant ratio $H_{s z} / h_{s z}=\gamma_{s z}=0.4$.

The cross-shore directed eddy kinetic energy flux from surf zone to inner shelf $F_{k^{\prime}}^{(s z)}$ is scaled as,

$$
F_{k^{\prime}}^{(s z)} \approx \varrho h_{s z} \text { r.m.s. }\left\{U_{s z}^{(r o t)}\right\}^{3}
$$

where r.m.s. $\left\{U_{s z}^{(r o t)}\right\}$ is the quadratic along-shore mean of barotropic rotational horizontal velocity, i.e. neglecting wave orbital and Stokes undertow velocities. The third moment of $U_{s z}^{(r o t)}$ is non-zero owing to strong, concentrated offshore flow and generally weaker and broader onshore flow, and approximated using r.m.s. $\left\{U_{s z}^{(r o t)}\right\}^{3}$. Based on two-dimensional TRC-resolving simulations, the cross-shore distribution of barotropic cross-shore exchange velocity is self-similar over a broad range of incident wave and beach slope variations, and well represented by Gaussian form,

$$
U_{E X}(x)=U_{0}^{(r o t)} \exp \left[-\frac{\left(x-x_{0}\right)^{2}}{L_{d}^{2}}\right],
$$

where $U_{0}^{(r o t)}$ is a rotational velocity scale, $x_{0}$ the location of maximum velocity and $L_{d}$ the offshore decay length scale (Suanda \& Feddersen 2015). The Gaussian form (6.6) also 


\section{D.J. Grimes and F. Feddersen}

parameterizes the distribution of r.m.s. $\left\{U_{s z}^{(r o t)}\right\}$ with dimensional coefficients,

$$
\begin{aligned}
U_{0}^{(r o t)} & =C_{4} \sigma_{\theta} \sqrt{g h_{s z}}\left[1+70 S_{s z}\right], \\
x_{0} & =-C_{5} \frac{h_{s z}}{\beta_{S Z}}\left[1+0.005 S_{s z}^{-1}\right], \\
L_{d} & =C_{6} \frac{h_{s z}}{\beta_{S Z}} \operatorname{Ir\sigma }_{\theta}^{-1 / 4}\left[1+22 S_{s z}^{1 / 2}\right],
\end{aligned}
$$

where wave directional spread $\sigma_{\theta}$ describes whether waves are long (small $\sigma_{\theta}$ ) or short crested (large $\sigma_{\theta}$ ), and Iribarren number $I r=\beta_{S Z} / S_{S z}^{1 / 2}$ relates the bottom slope to the wave steepness at breaking $S_{s z}=H_{s z} / L_{\infty}$. The modified non-dimensional coefficients $\left\{C_{4}, C_{5}, C_{6}\right\}=\{0.1284,0.2075,0.7092\}$ minimize the squared error between (6.6) and the ensemble average $F_{k^{\prime}}(x)$ and decently reproduce the modelled $F_{k^{\prime}}(x)$ magnitude and distribution (magenta, figure $6 c$ ).

The surf-zone and TRC mixing effects on the inner-shelf have functional dependence on the incident wave field. Focusing on the $F_{k^{\prime}}$ dependence on incident wave height and directional spread, i.e. constant period (and $L_{\infty}$ ) and beach slope $\beta_{S Z}$, relations (6.5)-(6.9) gives,

$$
d_{s} \propto\left(g^{3 / 2} N_{0}^{-2} \tau_{m i x}\right)^{1 / 4} \sigma_{\theta}^{3 / 4}\left(H_{s z}\right)^{5 / 8}\left[1+70 S_{s z}\right]^{3 / 4} \exp \left[-\frac{3}{4} H_{s z} \sigma_{\theta}^{1 / 2} \mathcal{F}\right],
$$

where function $\mathcal{F}\left(H_{S z}, L_{\infty}, \beta_{S Z}\right)$ varies weakly with $H_{s z}$. The wave height increases $d_{S}$ owing to an increase in $U_{0}^{(r o t)}, h_{s z}$ and $L_{d}$. Directional spread also increases $d_{s}$ due to larger $U_{0}^{(r o t)}$, but decreases the decay length scale $L_{d}$, i.e. the TRC region shrinks. The leading $H_{S z}$ dependence of $\exp \left[\mathrm{]}\right.$ in (6.10) is due to the numerator $\left(x-x_{0}\right)^{2} \propto H_{s z}^{2}$ and denominator $L_{d}^{2} \propto H_{s z}$, whereas the $\sigma_{\theta}^{1 / 2}$ dependence is due to $L_{d} \propto \sigma_{\theta}^{-1 / 4}$. As $L_{d}$ quantifies the eddy velocity cross-shore decay length scale, qualitatively similar mixing and circulation patterns are expected with 2-layer inner-shelf stratifications (Maxworthy \& Monismith 1988 ), provided the surface layer is shallower than the depth spanned by TRC eddies $\approx \beta_{S Z}\left(x_{0}+L_{d}\right)=3.3 \mathrm{~m}$. A constant slope $\beta_{S Z}=0.025$ was used here, but the Suanda $\&$ Feddersen (2015) TRC scaling applied to a range bottom slopes $\beta_{S Z}=0.02-0.06$, suggesting the $F_{k^{\prime}}^{(s z)}$ scaling (6.5) is robust. However, the sensitivity of $d_{s}$ to bottom slope requires further work, as both the rip-current eddy velocities and mixing-induced potential energy scalings (6.1-6.3) depend on $\beta_{S Z}$. Lastly, without a suitable scaling for $\tau_{\text {mix }}$, and its wave or beach slope dependence, (6.10) is incomplete but illustrates the relationship between strength of surf-zone-generated rip currents and the inner-shelf response. With constant $\tau_{\text {mix }}$ and modelled wave conditions $H_{s z}=1 \mathrm{~m}, T_{p}=10 \mathrm{~s}$ and $\sigma_{\theta}=10$ degrees, (6.10) implies that $\pm 25 \%$ variation in $H_{S z}$ induces a $\pm 18 \%$ change in $d_{s}$, to within $1 \%$ holding $\mathcal{F}$ constant. Alternatively, varying $\sigma_{\theta}$ by $\pm 25 \%$ induces $\pm 15 \%$ change in $d_{s}$.

\section{Summary}

Idealized simulations are analysed to determine the bulk (or mean) effects of TRC-enhanced mixing on inner-shelf stratification and circulation. Localized TRC-region-enhanced mixing cause modelled mean isotherms to broaden toward shore, forming a near-field inner-shelf overturning circulation that strengthens with $\mathrm{d} T_{0} / \mathrm{d} z$. Sloping isotherms also drive a far-field mean along-shore current that strengthens with 


\section{Self-similar inner-shelf response to transient rip currents}

$\mathrm{d} T_{0} / \mathrm{d} z$. Inner-shelf stratification and circulation are scaled using relatively simple energy arguments and existing scalings.

The surf-zone and TRC forcing supply the energy that drives mixing on the stratified inner shelf. Domain-integrated eddy kinetic energy equilibrates in $1 \mathrm{~h}$, and depth-integrated cross-shore eddy kinetic flux is independent of $\mathrm{d} T_{0} / \mathrm{d} z$ and decays to near zero within $4 L_{S Z}$. The inhomogeneous cross-shore distribution of TRC-induced mixing increases both the domain-integrated background and available potential energy. The potential energy growth rate increases with $\mathrm{d} T_{0} / \mathrm{d} z$ and becomes quasi-constant after $24 \mathrm{~h}$, with background potential energy accounting for approximately $90 \%$ of growth. The specific background potential energy vertical structure is characterized by the vertical length scale $d_{s}$, that is roughly constant over 24-48 h and which decreases weakly with increasing stratification. The mean available potential energy, responsible for driving inner-shelf mean circulation, also increases with $\mathrm{d} T_{0} / \mathrm{d} z$ and has two reservoirs above/below $z=-d_{s}$ with the depth-integrated available potential energy maximum near where the depth $h=2 d_{s}$. The energy based length scale $d_{s}$ provides the basis for inner-shelf stratification and circulation scalings.

Inner-shelf stratification and circulation response are self-similar and categorized into near-field and far-field dynamical regions separated where the depth $h=3 d_{s}$. In the near field, a quasi-steady mean 3-layer cross-shore exchange flow $\left\langle\bar{u}_{L}\right\rangle$ transports mixed fluid offshore sub-surface with reduced stratification $\langle\bar{N}\rangle / N_{0} \approx 1 / 2$. Non-dimensional $\left\langle\bar{u}_{L}\right\rangle / \mathcal{U}$ exchange flow profiles collapse with intrusive stratified gravity current scaling $\mathcal{U} \propto\left(d_{s} N_{0}\right)$ and vs non-dimensional depth $z / d_{s}$, indicating a self-similar dynamics analogous to deep-water localized mixing theory and experiment. The cross-shore exchange velocity also collapses using near-field non-dimensional cross-shore coordinate $\sim x / d_{s}$. Thus, near-field response scales with $N_{0}$ and $d_{s}$. Farther offshore $\left(h>3 d_{s}\right)$, the far-field intrusion mean isotherms broaden toward shore, and the mean horizontal stratification is consistent with a geostrophic isotherm slope scaling $\mathrm{d} z / \mathrm{d} x=f / N_{0}$. At the location of maximum $\langle\bar{v}\rangle$, non-dimensional $\langle\bar{v}\rangle / \mathcal{V}$ vertical profiles collapse using velocity scale $\mathcal{V} \propto N_{0}$ based on the thermal wind relation and vs non-dimensional depth $z / d_{s}$. The offshore decay of $\langle\bar{v}\rangle$ is also self-similar using far-field non-dimensional cross-shore coordinate $\sim x / L_{R}$, with Rossby deformation radius $L_{R} \sim d_{S} N_{0} / f$. Thus, $d_{S}$ is the fundamental length scale quantifying inner-shelf response to surf-zone and TRC mixing.

A scaling for length scale $d_{s}$ is made by relating the near-field-integrated potential energy change to the work against gravity performed by TRC-induced mixing, parameterized through the surf-zone to inner-shelf cross-shore kinetic energy flux. Assuming steady near-field mean potential energy, the domain-integrated potential energy growth rate is consistent with a near-field to far-field cross-shore buoyancy flux estimated from $d_{s}, N_{0}$ and $\mathcal{U}$. Using an idealized well-mixed wedge geometry, analytical scalings for the specific background and available potential energy are qualitatively consistent with modelled distributions. The leading-order depth-integrated available potential energy maximum $\left(\varrho d_{S}^{3} N_{0}^{2}\right)$ is consistent with the modelled $\mathrm{d} T_{0} / \mathrm{d} z$ dependence. The cross-shore distribution of depth-integrated available potential energy is qualitatively consistent with a transformation of the idealized well-mixed wedge to an offshore well-mixed intrusion with characteristic aspect ratio $\mathrm{d} z / \mathrm{d} x=f / N_{0}$ or $d_{s} / L_{R}$.

The mean (24-48 h) inner-shelf response to TRC-induced mixing is estimated by varying $\mathrm{d} T_{0} / \mathrm{d} z$ or $N_{0}$, keeping wave forcing constant. Results are extrapolated to varying incident wave and surf-zone characteristics assuming the strength and distribution of TRC energy determine the scales of inner-shelf response. Using a barotropic TRC induced surf-zone to inner-shelf eddy kinetic energy flux scaling, increasing wave height 


\section{D.J. Grimes and F. Feddersen}

and directional spread are expected to increase $d_{s}$ (and vice versa). The observed buoyancy-driven 3-layer cross-shore exchange flow is not limited to TRC-induced mixing, as the minimum fundamental requirement is vertical stratification and cross-shore inhomogeneous mixing. As various other coastal processes can induce inhomogeneous mixing, e.g. surface and internal tides, winds, nonlinear internal waves and bores, these results have broader relevance. As incident wave conditions are not typically steady, there is also potential for sub-tidal inner-shelf variability modulated by surf-zone processes.

Acknowledgements. The presented data are digitally archived at https://doi.org/10.6075/J05Q4TMR (Grimes \& Feddersen 2020). We thank S. N. Giddings, T. Zdyrski and 3 anonymous reviewers for thoughtful insight that improved this work. This work is dedicated to the memory of Nirnimesh 'Nirni' Kumar who pioneered and inspired our research.

Funding. This work was funded under the CSIDE grant by the National Science Foundation (NSF OCE-1459389), and student funding was provided through the NSF-GRFP. This work used the Extreme Science and Engineering Discovery Environment (XSEDE) Comet at SDSC through allocation TG-OCE180014, which is supported by National Science Foundation grant number ACI-1548562.

Declaration of interest. The authors report no conflict of interest.

Author ORCIDs.

(1) Derek J. Grimes http://orcid.org/0000-0001-5286-7819;

(1) Falk Feddersen http://orcid.org/0000-0002-5488-9074.

\section{Appendix A. Conceptual model of TRC-induced mixing and analytic sorted temperature profile}

Herein, we provide the derivation for the zero-crossing depth $d_{s}$ and the $E_{a}^{(z, t)}(x)$ scalings for the idealized scenarios in figure $13(a, b)$ used to contextualize the results. Consider a simplified along-shore $(y)$ uniform domain with constant bathymetric slope $h=-\beta x$ extending offshore to $x=-L_{x}$, such that the cross-shore domain width at each $z$-level is given by $\mathcal{L}(z)=L_{x}+z / \beta$ (figure 13a). The impact of surf-zone and TRC-induced eddy-driven mixing on the structure of available and background potential energy is illustrated by assuming the TRC-region mixing homogenizes temperature over $x>-L_{m i x}$ (figure 13a), with offshore depth $h_{\text {mix }}=\beta L_{\text {mix }}$. The well-mixed fluid within area $A_{\text {mix }}=$ $\beta L_{\text {mix }}^{2} / 2$ has initial temperature $T_{0}(z)(2.1)$, such that homogenizing results in uniform temperature, $T_{\text {mix }}$,

$$
\begin{aligned}
T_{\text {mix }} & =A_{\text {mix }}^{-1} \int_{-L_{\text {mix }}}^{0} \int_{-h(x)}^{0} T_{0}(z) \mathrm{d} z \mathrm{~d} x \\
& =T_{\text {ref }}-\frac{1}{3} \beta L_{\text {mix }} \frac{\mathrm{d} T_{0}}{\mathrm{~d} z}, \\
& \equiv T_{0}\left(-d_{s}\right)
\end{aligned}
$$

where $d_{s}=\beta L_{m i x} / 3$ is the depth where the initial $T_{0}(z)(2.1)$ matches the well-mixed fluid and is thus equivalent to the $\Delta T_{s}$ zero-crossing depth $d_{s}$ (figure 13a).

The sorted density and temperature profiles for the idealized wedge in figure $13(a)$ are determined by redistributing temperature above $z=-h_{\text {mix }}=-3 d_{s}$ such that all isotherms are horizontal and intersect the sloping bottom (cf. §4.2). After sorting, fluid initially within the region $x<-L_{\text {mix }}$ and $-3 d_{s}<z<-d_{s}$ vertically contracts to occupy $-3 d_{s}<$ $z<-d_{s}^{-}$, i.e. with $d_{s}^{-}>d_{s}$. The depth $d_{s}^{-}$is determined by equating the initial area 
offshore of $x=-L_{m i x}$ and in the range $-3 d_{s}<z-d_{s}$,

$$
A_{0}^{-}=2 d_{s}\left(L_{x}-L_{m i x}\right)
$$

to the sorted area occupying the full cross-shore domain $\mathcal{L}(z)$ over $-3 d_{s}<z<-d_{s}^{-}$,

$$
\begin{aligned}
A_{s}^{-} & =\int_{-3 d_{s}}^{-d_{s}^{-}} \mathcal{L}(z) \mathrm{d} z \\
& =\left(3 d_{s}-d_{s}^{-}\right) L_{x}+\left[\left(d_{s}^{-}\right)^{2}-9 d_{s}^{2}\right](2 \beta)^{-1},
\end{aligned}
$$

resulting in a quadratic equation for $d_{s}^{-}$, with the relevant root being,

$$
d_{s}^{-}=\beta L_{x}\left[1-\left(1-\epsilon_{\Delta}^{-}\right)^{1 / 2}\right]
$$

where the non-dimensional sorted layer thickness parameter is,

$$
\epsilon_{\Delta}^{-}=2 \epsilon_{z}+3 \epsilon_{z}^{2}
$$

with $\epsilon_{z}=d_{s} / h_{\max }$ (or $L_{\text {mix }} / L_{x}=3 \epsilon_{z}$ ) quantifying the relative size of zero-crossing depth to the maximum depth $h_{\max }=\left(\beta L_{x}\right)$. The modelled $\max \left\{d_{s}^{48 \mathrm{~h}}\right\}=4.3 \mathrm{~m}$ suggests $\epsilon_{z}<$ 0.04 for the present simulations. Expanding (A4) to $O\left(\epsilon_{z}\right)$ gives,

$$
d_{s}^{-} \sim d_{s}\left(1+2 \epsilon_{z}\right)
$$

Applying the same procedure to redistribute the stratified near-surface offshore region $x<-L_{m i x}$ and $z>-d_{s}$ to occupy the full cross-shore domain and $z>-d_{s}^{+}$, gives

$$
d_{s}^{+}=\beta L_{x}\left[1-\left(1-\epsilon_{\Delta}^{+}\right)^{1 / 2}\right],
$$

where,

$$
\epsilon_{\Delta}^{+}=2 \epsilon_{z}-6 \epsilon_{z}^{2}
$$

such that to $O\left(\epsilon_{z}\right)$,

$$
d_{s}^{+}=d_{s}\left(1-\frac{5}{2} \epsilon_{z}\right)
$$

Lastly, the well-mixed region $x>-L_{\text {mix }}$ spans $-d_{s}^{-}<z<-d_{s}^{+}$after sorting. It is worth noting that the separation between $\Delta T_{s}$ extrema scales as $\Delta d_{s}=9 / 2 d_{s} \epsilon_{z}$, or $\approx 0.18 d_{s}$, roughly $1 / 4$ the modelled separation $\Delta d_{s}^{48 \mathrm{~h}} \approx 0.75 d_{s}^{48 \mathrm{~h}}$.

Following the sorting procedure, isotherms from the stratified region offshore of $x<-L_{\text {mix }}$ become compressed, with $\partial T_{s} / \partial z \geq \mathrm{d} T_{0} / \mathrm{d} z$. The sorted temperature profile for upper and lower sections are derived by matching $T_{s}(0)=T_{0}(0)$ and $T_{s}\left(-3 d_{s}\right)=$ $T_{0}\left(-3 d_{s}\right)$ and conserving area between initial and sorted isotherms. At $z=\left\{-3 d_{s}, 0\right\}$, 


\section{D.J. Grimes and F. Feddersen}

the conservation of area between isotherms separated by,

$$
\Delta T=\Delta z \frac{\mathrm{d} T_{0}}{\mathrm{~d} z} \equiv \Delta z_{s} \frac{\partial T_{s}}{\partial z},
$$

requires the initial and sorted isotherm separations $\left\{\Delta z, \Delta z_{s}\right\}$ satisfy,

$$
\Delta z_{s} \mathcal{L}(z)=\Delta z\left(L_{x}-L_{m i x}\right)
$$

Substituting relation (A11) in (A10) gives,

$$
\frac{\partial T_{s}}{\partial z}=\frac{\mathrm{d} T_{0}}{\mathrm{~d} z}\left(1+\epsilon_{z} z / d_{s}\right) /\left(1-3 \epsilon_{z}\right),
$$

and $T_{s}$ is given in the upper and lower region by integration of (A12) with $T_{s}=T_{0}$ at $z=0$ and $z=-3 d_{s}$, respectively. Thus, the sorted temperature is piecewise continuous,

$$
T_{s}= \begin{cases}T_{r e f}+z\left(1+\epsilon_{z} z /\left(2 d_{s}\right)\right) \frac{\mathrm{d} T_{0}}{\mathrm{~d} z}\left(1-\epsilon_{x}\right)^{-1} & \text { for } z>-d_{s}^{+}, \\ T_{\text {mix }} & \text { for }-d_{s}^{-}<z<-d_{s}^{+}, \\ T_{\varnothing}+z\left(1+\epsilon_{z} z /\left(2 d_{s}\right)\right) \frac{\mathrm{d} T_{0}}{\mathrm{~d} z}\left(1-\epsilon_{x}\right)^{-1} & \text { for }-3 d_{s}<z<-d_{s}^{-}\end{cases}
$$

where $T_{r e f}=T_{0}(0)$ and $T_{\varnothing}=T_{0}\left(9 / 2 \epsilon_{z} d_{s} /\left(1-3 \epsilon_{z}\right)\right)$ satisfy the matching conditions. Thus, for small $d_{s}$ or large $h_{\max }$ the sorted temperature profile approaches the initial condition (2.1), i.e. $T_{s}(z)=T_{0}(z)$.

\section{Appendix B. Idealized available potential energy}

The specific available potential energy is determined using (4.8) by integrating from the reference level $z_{s}$ to $z$. In the well-mixed region $x>-L_{m i x}$, the sorted reference depth $z_{s}=-d_{s}^{ \pm}$and the displacement distance $\left|z-z_{s}\right|$ is linear. For $z>-d_{s}^{+}$,

$$
\begin{aligned}
\mathcal{E}_{a}^{+}(z) & =\varrho N_{0}^{2} d_{s} \int_{-d_{s}}^{z}\left[1+\left(\frac{z}{d_{s}}\right)\right] \mathrm{d} z,+O\left(\epsilon_{z}\right) \\
& =\frac{1}{2} \varrho N_{0}^{2} d_{s}^{2}\left[1+\left(\frac{z}{d_{s}}\right)\right]^{2}+O\left(\epsilon_{z}\right),
\end{aligned}
$$

and is equivalent to $\mathcal{E}_{a}^{-}(z)$ at leading order (right of figure 13b). The cross-shore varying $E_{a}^{z}(x)$ is given by combining the depth-integrated $\mathcal{E}_{a}^{ \pm}(z)$. Integrating $\mathcal{E}_{a}^{+}$over $z \geq-d_{s}^{+} \sim$ $-d_{s}$ gives,

$$
\begin{aligned}
E_{a}^{z^{+}}(x) & =\frac{1}{2} \varrho N_{0}^{2} d_{s}^{2} \int_{-\max \left\{d_{s}, h(x)\right\}}^{0}\left[1+\left(\frac{z}{d_{s}}\right)\right]^{2} \mathrm{~d} z+O\left(\epsilon_{z}\right), \\
& =\frac{1}{6} \varrho N_{0}^{2} d_{s}^{3}\left[1-\left(1-\frac{\max \left\{d_{s}, h(x)\right\}}{d_{s}}\right)^{3}\right]+O\left(\epsilon_{z}\right),
\end{aligned}
$$

and is separated into two regions at $x=-d_{s}^{+} / \beta$. For $x \leq-d_{s}^{+} / \beta$, at leading order, $E_{a}^{z^{+}}=$ $\varrho N_{0}^{2} d_{s}^{3} / 6$ is independent of $x$. Onshore of $x=-d_{s}^{+} / \beta, E_{a}^{z^{+}}(x)$ decreases cubically with 
decreasing water depth (top of figure $13 b$ ). Integrating $\mathcal{E}_{a}^{-}$from $z=-h(x)$ to $z=-d_{s}^{-} \sim$ $-d_{s}$ gives,

$$
E_{a}^{z^{-}}(x)=\frac{1}{6} \varrho N_{0}^{2} d_{s}^{3}\left(\frac{h(x)}{d_{s}}-1\right)^{3}+O\left(\epsilon_{z}\right),
$$

and $E_{a}^{z^{-}}$increases cubically with water depth. Combining the upper and lower layer $E_{a}^{z^{ \pm}}$ gives,

$$
E_{a}^{z} \approx \begin{cases}\frac{1}{2} \varrho N_{0}^{2} d_{s}^{3}\left[\left(\frac{h(x)}{d_{s}}\right)-\left(\frac{h(x)}{d_{s}}\right)^{2}+\frac{1}{3}\left(\frac{h(x)}{d_{s}}\right)^{3}\right] & \text { for } x>-d_{s}^{+} / \beta, \\ \frac{1}{6} \varrho N_{0}^{2} d_{s}^{3} & \text { for }-d_{s}^{-} / \beta<x<-d_{s}^{+} / \beta, \\ \frac{1}{6} \varrho N_{0}^{2} d_{s}^{3}\left[\left(\frac{h(x)}{d_{s}}\right)-1\right]^{3} & \text { for }-3 d_{s} / \beta<z<-d_{s}^{-} / \beta .\end{cases}
$$

Thus, $E_{a}^{z}(x)$ varies cubically in terms of $(-\beta x) / d_{s}$ outside of the narrow region $-d_{s}^{-} / \beta \leq$ $x \leq-d_{s}^{+} / \beta$ where $E_{a}^{z}$ is constant. Offshore of the well-mixed region, $x<-L_{\text {mix }}, \mathcal{E}_{a}^{ \pm}$are $O\left(\epsilon_{z}^{2}\right)$, implying that the offshore area-integrated $E_{a}^{(x, z)}$ is $O\left(\epsilon_{z}\right)$. Hereafter, we neglect the offshore region. Cross-shore integrating $E_{a}^{z}(x)$ gives, $E_{a}^{\left(x, z^{+}\right)}+E_{a}^{\left(x, z^{-}\right)}=\varrho N_{0}^{2} d_{s}^{4} / \beta[1 / 8+$ $1 / 3+2 / 3]=E_{p}^{\text {mix }} / 2$.

To compare the idealized cross-shore varying $E_{a}^{z}(x)$ to the modelled $E_{a}^{(z, t)}\left(x, \mathrm{~d} T_{0} / \mathrm{d} z\right)$, the vertical front at $x=-L_{m i x}$ in figure 13(a) is transformed into sloping fronts above and below $z=-d_{s}$ in figure $13(b)$, qualitatively consistent with model sloping far-field $\langle\bar{T}\rangle$ isotherms (figure $3 d-f$ ). The depth of upper and lower sloping fronts are,

$$
H^{ \pm}=d_{s} \mp \beta_{f}^{ \pm}\left(x+L_{f}\right),
$$

with $H^{+}=H^{-}$at the offshore apex located at $x=-L_{f}$ (figure 13b). Upper/lower frontal slopes $\beta_{f}^{ \pm}$may differ from one another to satisfy the dynamics or conservation relations (e.g. conserve area, mean temperature or potential energy) or enforce that surface/bottom fronts occur offshore of the TRC region $\left(x>-4 L_{S Z}\right)$ or surf zone $\left(x>-L_{S Z}\right)$. For symmetric upper/lower frontal slope $\beta_{f}$, the idealized intrusion has vertical thickness,

$$
H(x)=H^{-}(x)-H^{+}(x)=2 \beta_{f}\left(x+L_{f}\right),
$$

varying with offshore length scale $L_{f}$ and the frontal slope $\beta_{f}$. For simplicity, the values used in figure 13(b) were determined by constraining the offshore apex depth to $z=-d_{s}$ while conserving the initial area $A_{m i x}=(9 / 2) d_{s}^{2} / \beta_{S Z}$. Thus, figure $13(b)$ is an adiabatic transformation of figure 13(b), neglecting the adjustment of isotherms initially offshore of $x=-L_{\text {mix }}$. Alternative constraints, e.g. conserving the well-mixed fluid temperature, further complexify the derivation. As the adjustment does not conserve potential energy or potential vorticity, it is only meant as a qualitative illustration of the cross-shore $E_{a}^{z}$ structure. For brevity, derivation of $L_{f}$ is only summarized. Integrating over the well-mixed intrusion and wedge of figure $13(b)$ and equating to $A_{\text {mix }}$ results in the quadratic 


\section{D.J. Grimes and F. Feddersen}

polynomial,

$$
a L_{f}^{2}+b L_{f}-c=0
$$

with variable coefficients,

$$
\begin{aligned}
& a=\beta_{f}^{2} \beta, \\
& b=2 d_{s} \beta_{f} \beta, \\
& c=d_{s}^{2}\left(2 \beta_{f}+\beta\right)+9 d_{s}^{2} \beta_{f}\left(\beta_{f}+\beta\right) / \beta,
\end{aligned}
$$

and taking the positive root gives,

$$
L_{f}=\frac{-b+\sqrt{b^{2}+4 a c}}{2 a} .
$$

The relevant result being that the cross-shore length scale $L_{f}$ is inversely proportional to $\beta_{f}$, i.e. smaller $\beta_{f}$ induces larger $L_{f}$. For very small $\beta_{f}$, enforcing conservation of area can lead to the surface front intersecting the surf zone $x=-L_{S Z}$; this may be avoided by modifying $\beta_{f}^{ \pm}$.

Based on the leading-order quadratic dependence $\mathcal{E}_{a}^{ \pm}$(B1), the depth-integrated $E_{a}^{z}(x)$ (B4) varies cubically in $(-\beta x) / d_{s}$. Similarly, in the transformed configuration in figure 13(b) the far-field region where well-mixed fluid is bounded above and below by sloping $H^{ \pm}$(offshore of triangle, figure $13 b$ ), $E_{a}^{z}$ also varies cubically. Specifically, moving offshore from $x=0$ to the $E_{a}^{z}$ maximum, which occurs where the lower sloping front $H^{-}$intersects the bottom (square, figure 13b), $E_{a}^{z}$ is described by (B4). Offshore of the maximum, the depth integrated $E_{a}^{z}(x)$ is given by,

$$
E_{a}^{z} \approx \begin{cases}\frac{1}{6} \varrho N_{0}^{2} d_{s}^{3}\left[1+\left(\frac{\beta_{f}}{d_{s}}\right)^{3}\left(x+L_{f}\right)^{3}\right] & \text { for } \Delta<x<\square, \\ \frac{1}{3} \varrho N_{0}^{2} d_{s}^{3}\left(\frac{\beta_{f}}{d_{s}}\right)^{3}\left(x+L_{f}\right)^{3} & \text { for } x<\Delta\end{cases}
$$

where $x$ ranges correspond to markers in figure 13(b). For $x<\Delta$, shifting the origin to the surface front at $x=\triangle=x_{0}$, where $\left(x+L_{f}\right)=\left(x-x_{0}+d_{s} / \beta_{f}\right)$, and substituting $\beta_{f}=$ $d_{s} / L_{R} \approx f / N_{0}$ gives a far-field scaling,

$$
E_{a}^{(z, f f)}(x)=\frac{1}{3} \varrho N_{0}^{2} d_{s}^{3}\left[1+L_{R}^{-1}\left(x-x_{0}\right)\right]^{3} .
$$

\section{Appendix C. Idealized background potential energy}

The change in background potential energy is determined using (4.7) with the leading-order $T_{s}(z)$ (A13). The vertical distribution of specific background potential energy change is piecewise continuous with,

$$
\Delta \mathcal{E}_{b}(z)= \begin{cases}-\varrho N_{0}^{2} C_{\epsilon} z^{2}\left[1+z /\left(6 d_{s}\right)\right] & \text { for } z>-d_{s}^{+}, \\ \varrho N_{0}^{2} z\left(z+d_{s}\right) & \text { for }-d_{s}^{-}<z<-d_{s}^{+}, \\ -\varrho N_{0}^{2} C_{\epsilon} z\left[\frac{3}{2} d_{s}+z\left(1+z /\left(6 d_{s}\right)\right)\right] & \text { for }-3 d_{s}<z<d_{s}^{-},\end{cases}
$$


where $C_{\epsilon}=3 \epsilon_{z} /\left(1-3 \epsilon_{z}\right)$. Although the upper and lower regions $\Delta \mathcal{E}_{b}$ are $O\left(\epsilon_{z}\right)$, the area-integrated change is $O(1)$. The leading-order $z>-d_{s}^{+}$area-integrated $\Delta \mathcal{E}_{b}$ is,

$$
\begin{aligned}
E_{b}^{\left(x, z^{+}\right)} & =-\varrho N_{0}^{2}\left(3 \epsilon_{z} L_{x}\right) d_{s}^{2} \int_{-d_{s}}^{0}\left[\left(\frac{z}{d_{s}}\right)^{2}+\frac{1}{6}\left(\frac{z}{d_{s}}\right)^{3}\right] \mathrm{d} z+O\left(\epsilon_{z}\right), \\
& =-\frac{7}{24} \varrho N_{0}^{2} L_{m i x} d_{s}^{3}+O\left(\epsilon_{z}\right),
\end{aligned}
$$

where the substitution $L_{\text {mix }}=3 \epsilon_{z} L_{x}$ was made from line 1-2. The negative sign indicates that the sorted background potential energy is more negative than the initial potential energy, i.e. denser fluid at same $z$-level relative to the initial density profile. To leading order in the lower region,

$$
\begin{aligned}
E_{b}^{\left(x, z^{-}\right)} & =-\varrho N_{0}^{2} L_{m i x} d_{s}^{2} \int_{-3 d_{s}}^{-d_{s}}\left[\frac{3}{2}\left(\frac{z}{d_{s}}\right)+\left(\frac{z}{d_{s}}\right)^{2}+\frac{1}{6}\left(\frac{z}{d_{s}}\right)^{3}\right] \mathrm{d} z+O\left(\epsilon_{z}\right), \\
& =\frac{2}{3} \varrho N_{0}^{2} L_{m i x} d_{s}^{3}+O\left(\epsilon_{z}\right),
\end{aligned}
$$

and is $>0$ due to a deepening in the sorted isotherms, i.e. lighter water at depth. Over the unstratified region $-d_{s}^{-}<z<-d_{s}^{+}, E_{b}^{(x, z)}$ is $O\left(\epsilon_{z}\right)$ and tends to zero with increasing $L_{x}$. Thus, for a relatively large domain, the total change is $E_{b}^{(x, z)}=E_{p}^{\text {mix }} / 2$ and the potential energy change is equipartitioned between background and available constituents.

\section{REFERENCES}

Boehm, A.B., Ismail, N.S., Sassoubre, L.M. \& Andruszkiewicz, E.A. 2017 Oceans in peril: grand challenges in applied water quality research for the 21st century. Environ. Engng Sci. 34 (1), 3-15.

Bolster, D., Hang, A. \& Linden, P.F. 2008 The front speed of intrusions into a continuously stratified medium. J. Fluid Mech. 594, 369-377.

Booij, N., Ris, R.C. \& Holthuijsen, L.H. 1999 A third-generation wave model for coastal regions: 1. Model description and validation. J. Geophys. Res.: Oceans 104 (C4), 7649-7666.

BRINK, K.H. 2016 Cross-shelf exchange. Annu. Rev. Mar. Sci. 8 (1), 59-78.

Brown, J., MACMAhan, J., Reniers, A. \& ThORnton, E. 2009 Surf zone diffusivity on a rip-channeled beach. J. Geophys. Res.: Oceans 114, C11015.

BuRChARD, H. \& Bolding, K. 2001 Comparative analysis of four second-moment turbulence closure models for the oceanic mixed layer. J. Phys. Oceanogr. 31 (8), 1943-1968.

Castelao, R., Chant, R., Glenn, S. \& Schofield, O. 2010 The effects of tides and oscillatory winds on the subtidal inner-shelf cross-shelf circulation. J. Phys. Oceanogr. 40 (4), 775-788.

Castelle, B., Scott, T., Brander, R.W. \& McCarroll, R.J. 2016 Rip current types, circulation and hazard. Earth-Sci. Rev. 163, 1-21.

CheOng, H.-B., KuEnEn, J.J.P. \& Linden, P.F. 2006 The front speed of intrusive gravity currents. J. Fluid Mech. 552, 1-11.

Clark, D.B., Feddersen, F. \& GuZa, R.T. 2010 Cross-shore surfzone tracer dispersion in an alongshore current. J. Geophys. Res.: Oceans 115 (C10), c10035.

Clark, D.B., Feddersen, F. \& Guza, R.T. 2011 Modeling surf zone tracer plumes: 2. Transport and dispersion. J. Geophys. Res.: Oceans 116 (C11), c11028.

Dalrymple, R.A., MacMahan, J.H., Reniers, A.J.H.M. \& Nelko, V. 2011 Rip currents. Annu. Rev. Fluid Mech. 43 (1), 551-581.

Davies, P.A., Fernando, H.J.S., Besley, P. \& Simpson, R.J. 1991 Generation and spreading of a turbulent mixed layer in a rotating, stratified fluid. J. Geophys. Res.: Oceans 96 (C7), 12567-12585.

Elgar, S. \& Raubenheimer, B. 2020 Field evidence of inverse energy cascades in the surfzone. J. Phys. Oceanogr. 50 (8), 2315-2321.

FEDDERSEN, F. 2012 Scaling surf zone turbulence. Geophys. Res. Lett. 39, L18613. 


\section{D.J. Grimes and F. Feddersen}

FEDdERSEN, F. 2014 The generation of surfzone eddies in a strong alongshore current. J. Phys. Oceanogr. 44 (2), 600-617.

Feddersen, F., MacMahan, J.H., Freismuth, T.M., Gough, M.K. \& Kovatch, M. 2020 Inner-shelf vertical and alongshore temperature variability in the subtidal, diurnal, and semidiurnal bands along the central california coastline with headlands. J. Geophys. Res.: Oceans 125, e2019JC015347.

Garrett, C.J. \& Loder, J.W. 1981 Dynamical aspects of shallow sea fronts. Phil. Trans. R. Soc. Lond. A302, 563-581.

GregG, M.C., D’Asaro, E.A., Riley, J.J. \& Kunze, E. 2018 Mixing efficiency in the ocean. Annu. Rev. Mar. Sci. 10 (1), 443-473.

GRIMES, D.J. \& FEDDERSEN, F. 2020 Data from: the self-similar stratified inner-shelf response to transient rip-current induced mixing. In Transient Rip-Currents on the Stratified Inner-shelf . UC San Diego Library Digital Collections.

Grimes, D.J., Feddersen, F., Giddings, S.N. \& Pawlak, G. 2020a Cross-shore deformation of a surfzone-released dye plume by an internal tide on the inner shelf. J. Phys. Oceanogr. 50 (1), 35-54.

Grimes, D.J., FEDdERSEN, F. \& KuMAR, N. $2020 b$ Tracer exchange across the stratified inner-shelf driven by transient rip-currents and diurnal surface heat fluxes. Geophys. Res. Lett. 47, e2019GL086501.

Hally-Rosendahl, K., Feddersen, F., Clark, D.B. \& GuZA, R.T. 2015 Surfzone to inner-shelf exchange estimated from dye tracer balances. J. Geophys. Res.: Oceans 120 (9), 6289-6308.

Hally-Rosendahl, K., Feddersen, F. \& GuZA, R.T. 2014 Cross-shore tracer exchange between the surfzone and inner-shelf. J. Geophys. Res.: Oceans 119 (7), 4367-4388.

VAN HEIJST, G.J.F 1986 On the dynamics of a tidal mixing front. In Marine Interfaces Ecohydrodynamics (ed. J.C.J. Nihoul), Elsevier Oceanography Series, vol. 42, pp. 165-194. Elsevier.

Hopfinger, E.J. \& TOLY, J.-A. 1976 Spatially decaying turbulence and its relation to mixing across density interfaces. J. Fluid Mech. 78 (1), 155-175.

Johnson, D. \& Pattiaratchi, C. 2006 Boussinesq modelling of transient rip currents. Coast. Engng 53 (5), 419-439.

KANTHA, L.H. \& Clayson, C.A. 1994 An improved mixed layer model for geophysical applications. J. Geophys. Res.: Oceans 99 (C12), 25235-25266.

Kumar, N. \& Feddersen, F. 2017a The effect of Stokes drift and transient rip currents on the inner shelf. Part I: no stratification. J. Phys. Oceanogr. 47 (1), 227-241.

Kumar, N. \& Feddersen, F. $2017 b$ The effect of Stokes drift and transient rip currents on the inner shelf. Part II: with stratification. J. Phys. Oceanogr. 47 (1), 243-260.

KumAR, N. \& FEDdERSEN, F. 2017c A new offshore transport mechanism for shoreline-released tracer induced by transient rip currents and stratification. Geophys. Res. Lett. 44, 2843-2851.

Lemagie, E.P. \& LerCZAK, J.A. 2015 A comparison of bulk estuarine turnover timescales to particle tracking timescales using a model of the yaquina bay estuary. Estuar. Coast. 38 (5), 1797-1814.

LENTZ, S.J. 2001 The influence of stratification on the wind-driven cross-shelf circulation over the North Carolina shelf. J. Phys. Oceanogr. 31 (9), 2749-2760.

LENTZ, S.J. \& CHAPMAN, D.C. 2004 The importance of nonlinear cross-shelf momentum flux during wind-driven coastal upwelling*. J. Phys. Oceanogr. 34 (11), 2444-2457.

LentZ, S.J. \& Fewings, M.R. 2012 The wind- and wave-driven inner-shelf circulation. Annu. Rev. Mar. Sci. 4 (1), 317-343.

Lentz, S.J., Fewings, M., Howd, P., Fredericks, J. \& Hathaway, K. 2008 Observations and a model of undertow over the inner continental shelf. J. Phys. Oceanogr. 38 (11), 2341-2357.

MACCREAdY, P. \& GiddingS, S.N. 2016 The mechanical energy budget of a regional ocean model. J. Phys. Oceanogr. 46 (9), 2719-2733.

MacMahan, J., et al. 2010 Mean lagrangian flow behavior on an open coast rip-channeled beach: a new perspective. Mar. Geol. 268 (1), 1-15.

MAderich, V.S., VAN HeiJst, G.J.F. \& BRANDT, A. 2001 Laboratory experiments on intrusive flows and internal waves in a pycnocline. J. Fluid Mech. 432, 285-311.

MANins, P.C. 1976 Intrusion into a stratified fluid. J. Fluid Mech. 74 (3), 547-560.

Maxworthy, T. \& Monismith, S.G. 1988 Differential mixing in a stratified fluid. J. Fluid Mech. 189, 571-598.

MEI, C.C 1992 The Applied Dynamics of Ocean Surface Waves. World Scientific.

Molina, L., Pawlak, G., Wells, J.R., Monismith, S.G. \& Merrifield, M.A. 2014 Diurnal cross-shore thermal exchange on a tropical forereef. J. Geophys. Res.: Oceans 119 (9), 6101-6120.

Monismith, S.G., Genin, A., Reidenbach, M.A., Yahel, G. \& Koseff, J.R. 2006 Thermally driven exchanges between a coral reef and the adjoining ocean. J. Phys. Oceanogr. 36 (7), 1332-1347. 
Morgan, S.G., Shanks, A.L., Macmahan, J.H., Reniers, A.J.H.M. \& Feddersen, F. 2018 Planktonic subsidies to surf-zone and intertidal communities. Annu. Rev. Mar. Sci. 10 (1), null.

Omand, M.M., Leichter, J.J., Franks, P.J.S., Guza, R.T., Lucas, A.J. \& Feddersen, F. 2011 Physical and biological processes underlying the sudden surface appearance of a red tide in the nearshore. Limnol. Oceanogr. 56 (3), 787-801.

Peregrine, D.H. 1998 Surf zone currents. Theor. Comput. Fluid Dyn. 10 (1), 295-309.

Reniers, A.J.H.M., MacMahan, J.H., Beron-Vera, F.J. \& Olascoaga, M.J. 2010 Rip-current pulses tied to lagrangian coherent structures. Geophys. Res. Lett. 37 (5).

RodrigueZ, A.R., GiddingS, S.N. \& KUMAR, N. 2018 Impacts of nearshore wave-current interaction on transport and mixing of small-scale buoyant plumes. Geophys. Res. Lett. 45 (16), 8379-8389.

ShCHEPETKin, A.F \& MCWilliams, J.C 2005 The regional oceanic modeling system (roms): a split-explicit, free-surface, topography-following-coordinate oceanic model. Ocean Model. 9 (4), 347-404.

Sinnett, G., Feddersen, F., Lucas, A.J., Pawlak, G. \& Terrill, E. 2018 Observations of nonlinear internal wave run-up to the surfzone. J. Phys. Oceanogr. 48 (3), 531-554.

SuANDA, S.H. \& FedDERSEN, F. 2015 A self-similar scaling for cross-shelf exchange driven by transient rip currents. Geophys. Res. Lett. 42 (13), 5427-5434.

Thomas, P.J. \& Linden, P.F. 2007 Rotating gravity currents: small-scale and large-scale laboratory experiments and a geostrophic model. J. Fluid Mech. 578, 35-65.

Walter, R.K., Woodson, C.B., Arthur, R.S., Fringer, O.B. \& Monismith, S.G. 2012 Nearshore internal bores and turbulent mixing in southern monterey bay. J. Geophys. Res.: Oceans 117 (C7), c07017.

Warner, J.C, Armstrong, B., He, R. \& Zambon, J.B 2010 Development of a coupled ocean-atmosphere-wave-sediment transport (COAWST) modeling system. Ocean Model. 35 (3), 230-244.

WARNER, J.C., GEYER, W.R. \& LERCZAK, J.A. 2005 Numerical modeling of an estuary: a comprehensive skill assessment. J. Geophys. Res.: Oceans 110 (C5).

WASHBURn, L. \& MCPHEE-SHAw, E. 2013 Coastal transport processes affecting inner-shelf ecosystems in the California current system. Oceanography 26 (3), 34-43.

White, B.L. \& HeLFRICH, K.R. 2008 Gravity currents and internal waves in a stratified fluid. J. Fluid Mech. $616,327-356$.

Winters, K.B. \& BARKAN, R. 2013 Available potential energy density for boussinesq fluid flow. J. Fluid Mech. 714, 476-488.

Winters, K.B., Lombard, P.N., Riley, J.J. \& D'Asaro, E.A. 1995 Available potential energy and mixing in density-stratified fluids. J. Fluid Mech. 289, 115-128.

YU, J. \& SLINN, D.N. 2003 Effects of wave-current interaction on rip currents. J. Geophys. Res.: Oceans 108 (C3). 